\title{
Adverse events of nucleos(t)ide analogues for chronic hepatitis B: a systematic review
}

\author{
Raquel Scherer de Fraga ${ }^{1,3}$ - Victor Van Vaisberg ${ }^{1}$ Luiz Cláudio Alfaia Mendes ${ }^{2}$ • \\ Flair José Carrilho ${ }^{1,2} \cdot$ Suzane Kioko Ono ${ }^{1,2}$
}

Received: 1 July 2019/Accepted: 26 February 2020/Published online: 17 March 2020

(C) The Author(s) 2020

\begin{abstract}
Nucleos(t)ide analogues (NAs) are the main drug category used in chronic hepatitis B (CHB) treatment. Despite the fact that NAs have a favourable safety profile, undesired adverse events (AEs) may occur during the treatment of CHB. Given the eminent number of patients currently receiving NAs, even a small risk of any of these toxicities can represent a major medical issue. The main objective of this review was to analyse information available on AEs associated with the use of NAs in published studies. We choose the following MesH terms for this systematic review: chronic hepatitis B, side effects and treatment. All articles published from 1 January 1990 up to 19 February 2018 in MEDLINE of PubMed, EMBASE, the Cochrane Library and LILACS databases were searched. A total of 120 articles were selected for analysis, comprising 6419 patients treated with lamivudine (LAM), 5947 with entecavir (ETV), 3566 with tenofovir disoproxil fumarate (TDF), 3096 with telbivudine (LdT), 1178 with adefovir dipivoxil (ADV) and 876 with tenofovir alafenamide (TAF). The most common AEs in all NAs assessed were abdominal pain/discomfort, nasopharyngitis/upper respiratory tract infections, fatigue, and headache. TAF displays the highest density of AEs per patient treated among NAs
\end{abstract}

Raquel Scherer de Fraga

rschfraga@gmail.com

1 Department of Gastroenterology, University of São Paulo School of Medicine, Av. Dr. Enéas Carvalho de Aguiar, 255 ICHC, 9th floor, Office 9159, São Paulo, SP 05403-000, Brazil

2 Division of Clinical Gastroenterology and Hepatology, Hospital das Clínicas, University of São Paulo School of Medicine, São Paulo, SP, Brazil

3 IMED School of Medicine, Passo Fundo, RS, Brazil
(1.14 AE/treated patient). In conclusion, treatment of CHB with NAs is safe, with a low incidence of AEs. Despite the general understanding TAF being safer than TDF, the number of patients treated with TAF still is too small in comparison to other NAs to consolidate an accurate safety profile. PROSPERO Registration No. CRD42018086471

Keywords Chronic hepatitis B · Nucleotide/nucleoside analogues · Adverse events

\section{Introduction}

An estimated 257 million people globally are living with chronic hepatitis B (CHB) infection, according to the World Health Organization in 2018 [1]. Treatment's main goals in CHB are to halt disease progression and prevent disease-related complications, achieved by suppression of hepatitis B virus (HBV) DNA replication [2]. To the present date, CHB treatment is either based on nucleos(t)ide analogue (NA) or on interferon IFN $\alpha$, currently pegylated $(\mathrm{PegIFN} \alpha)$ [3, 4]. NAs that have been approved for HBV treatment in humans include lamivudine (LAM), adefovir dipivoxil (ADV), entecavir (ETV), telbivudine (LdT), tenofovir disoproxil fumarate (TDF) and tenofovir alafenamide (TAF), and can be classified into those associated with low barrier against HBV resistance (LAM, ADV, LdT) and those with high barrier to HBV resistance (ETV, TDF, TAF) [3-5]. The main advantage of treatment with a potent NA with high barrier to resistance (i.e., ETV, TDF, $\mathrm{TAF}$ ), considered to be the first-line treatment for CHB, is its predictable high long-term antiviral efficacy leading to undetectable HBV DNA levels in the vast majority of compliant patients as well as its good safety profile [3-5]. Moreover, it has been shown that NAs can improve the 
liver fibrosis and reduce the hepatocarcinogenesis in patients with CHB [6-8].

A significant number of patients has been treated with NAs to date, having increased the experience with their efficacy, resistance and safety profile over the years. Despite the fact that NAs have a favourable safety profile [3, 4], undesired adverse events (AEs) may occur during the treatment of CHB infection. Given the eminent number of patients currently receiving NAs, even a small risk of any of these toxicities can be translated into a major medical issue.

The main objective of this systematic review is to analyse available information in published studies on AEs associated with Nas' use in adults.

\section{Methods}

\section{Eligibility criteria}

The following research questions were addressed:

- What are the most common AEs with the use of NAs in the CHB treatment?

- Is there any difference in the incidence of AEs between the different NAs?

- Do patients receiving TAF have fewer AEs compared to TDF?

A PICO model was constructed (participants, interventions, control and outcome):

\section{Participants}

- Adults $>18$ years old diagnosed with HBV infection.

\section{Interventions}

- Antiviral therapy with NAs (LAM, ADV, LdT, ETV, TDF or TAF).

\section{Control}

- We used only the data for the currently approved dose, i.e. LAM 100 or 150 mg; ADV 10 mg; LdT 600 mg; ETV 0,5 or $1,0 \mathrm{mg}$; TDF $300 \mathrm{mg}$; TAF $25 \mathrm{mg}$.

- Studies based only on drug-combination regimens were excluded due to difficulties in evaluating cause-effect relationship.

- Studies with both single drug arm and drug-combinations arm; only the single drug arm were included in the analysis.
Outcome measure

- Adverse events (AEs).

\section{Exclusion criteria}

We excluded studies whose patients presented acute HBV infection, acute liver failure, decompensated cirrhosis, pregnancy, hepatitis $\mathrm{C}$ or $\mathrm{D}$ or human immunodeficiency virus (HIV) coinfection, schistosomiasis infection; patients receiving corticosteroids, chemotherapy, or immunosuppressive therapy; transplant recipients; and hemodialysis patients. Likewise, studies that did not report AEs or stated "no serious adverse events" or "no significant difference in side effects between groups" with no further AEs description were excluded.

\section{Literature search strategy}

This study was performed according to the PRISMA statement [9]. We chose the following MesH terms: chronic hepatitis $\mathrm{B}$, side effects and treatment. We reviewed all articles published from 1 January 1990 up to 19 February 2018 in MEDLINE of PubMed, EMBASE, the Cochrane Library and LILACS databases and included studies published in English language. Since the NAs have a good safety profile with a small percentage of AEs, we enrolled both observational (i.e. cohort, case-control and cases series) and randomized controlled trials (RCTs) as a search strategy for maximizing AEs sensitivity. All the references identified were managed by Endnote. The flowchart in Fig. 1 shows the process of review of publications. We followed an established protocol which had been registered in PROSPERO (International prospective register of systematic reviews) [10], and the record is available on https://www.crd.york.ac.uk/prospero/ (Registration No. CRD42018086471).

\section{Data collection and quality assessment}

The following data were extracted from included studies: study design, country where the study was conducted, first author, publication year, number of participants, inclusion and exclusion criteria, drug dosing regimens and AEs reported. Two reviewers independently performed data extraction (RSF and VVV) and discrepancies were discussed during a consensus meeting.

To facilitate data analysis, AEs were divided into groups, similar to those found in the VigiAccess ${ }^{\mathrm{TM}}$ database, as follows: blood and lymphatic system disorders; cardiac disorders; ear and labyrinth disorders; endocrine disorders; eye disorders; gastrointestinal disorders; general 
Figure1 Flowchart of study selection. MEDLINE Medical Literature Analysis and Retrieval System Online, EMBASE Excerpta Medica Database, Cochrane

The Cochrane Library, LILACS Literatura Latino-Americana e do Caribe em Ciências da Saúde, $A E s$ adverse events, $T D F$ tenofovir disoproxil fumarate, $L d T$ telbivudine

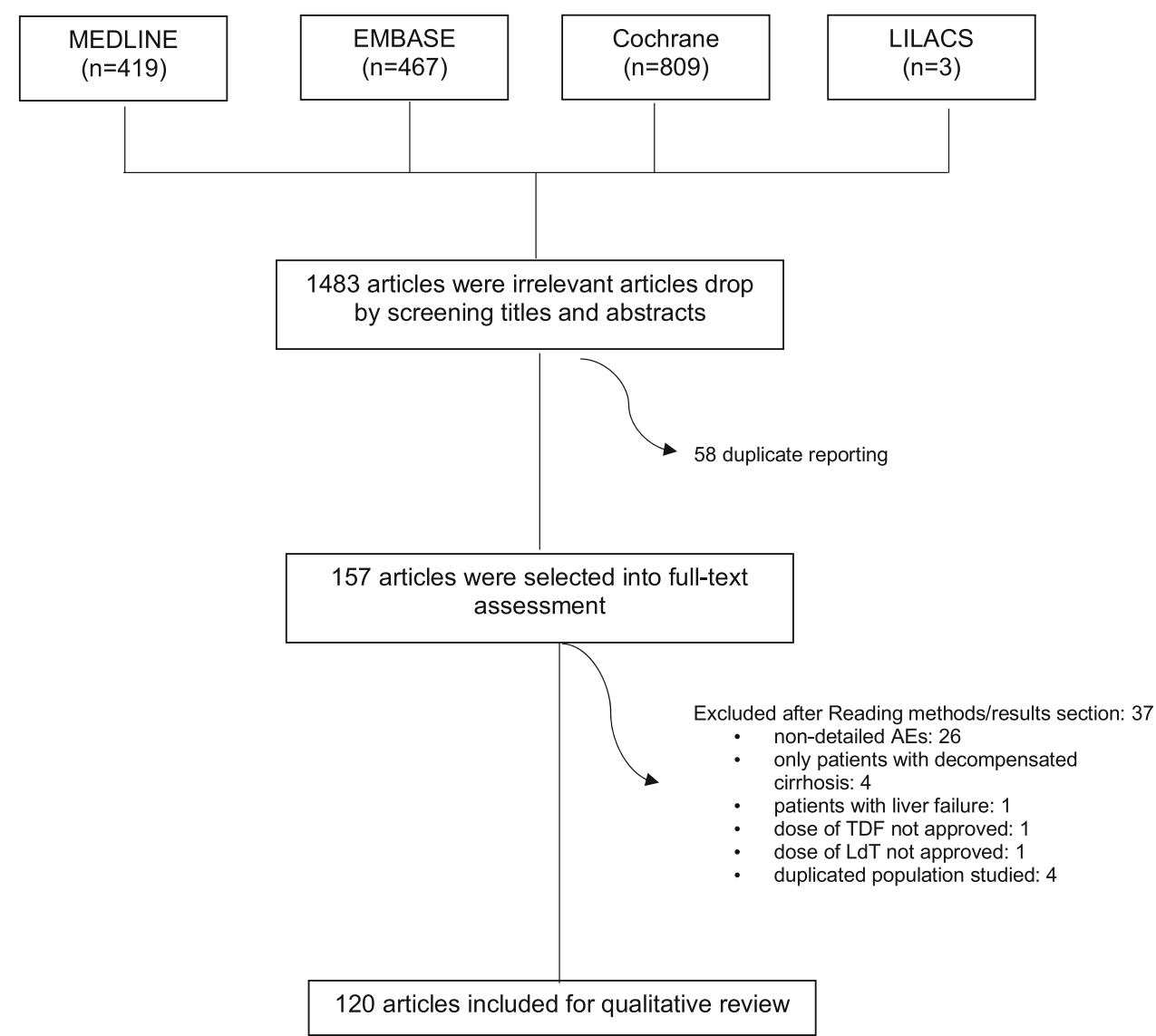

disorders; hepatobiliary disorders; infections and infestations; laboratory abnormalities; metabolism and nutrition disorders; musculoskeletal and connective tissue disorders; neoplasms; nervous system disorders; psychiatric disorders; renal and urinary disorders, reproductive system disorders; respiratory disorders; skin and subcutaneous tissue disorders.

\section{Results}

\section{Studies}

A total of 1698 articles were retrieved. Two authors conducted an initial screening and 1483 studies were excluded after reading titles and abstracts. Following the removal of duplicates, 157 full-text articles were assessed for eligibility. Thirty-seven studies were excluded for the following reasons: non-detailed adverse events (26), only patients with decompensated cirrhosis (4), patients with liver failure (1), dose of TDF not approved (1), dose of LdT not approved (1) and duplicated population studied (4). Finally, 120 articles were selected for analysis. The listing of the included reporters and their characteristics are shown in Table 1.
There were 6419 patients treated with LAM, 5947 treated with ETV, 3566 treated with TDF, 3096 treated with LdT, 1178 treated with ADV and 876 treated with TAF.

Table 2 contains the AEs described in the studies, depending on the drugs used.

Neoplasms were documented in 39 patients, with hepatocellular carcinoma being the most frequent $-67 \%$ ( $n$. 26: LAM-8; ETV-9; TDF-3; LdT-6) [Table 2]. None of the cases were related to NAs use.

\section{Lamivudine (LAM)}

In studies using $100 \mathrm{mg}$ of LAM, a total of 5554 AEs were reported (0.87 AE/patient treated) [Fig. 2, Table 2].

The most frequent AEs reported were gastrointestinal disorders (20.1\%), infections and infestations (15.7\%), general disorders $(14.6 \%)$, respiratory disorders $(12.5 \%)$ and nervous system disorders (12\%) [Table 2].

Among gastrointestinal events, the most reported were abdominal pain or discomfort $(n=411)$.

The most commonly described infections were upper respiratory tract infection $(n=413)$.

General disorders included nonspecific symptoms, with asthenia/fatigue being the most reported $(n=672)$. 
Table 1 Studies reported in this review

\begin{tabular}{|c|c|c|c|c|}
\hline Authors, year & Country & Patients $(n)$ & Drugs & Study design \\
\hline $\begin{array}{l}\text { Koike, } 2018 \\
\quad[39]\end{array}$ & Japan & $\begin{array}{l}110 \\
56\end{array}$ & $\begin{array}{l}\text { TDF } \\
\text { ETV }\end{array}$ & $\begin{array}{l}\text { Randomized, active controlled, double- } \\
\text { blind, double-dummy, parallel arm } \\
\text { comparation }\end{array}$ \\
\hline An, 2017 [40] & Republic of Korea & $\begin{array}{l}47 \\
50\end{array}$ & $\begin{array}{l}\text { ETV } \\
\text { LdT }\end{array}$ & Randomized open-label \\
\hline $\begin{array}{l}\text { Ashgar, } 2017 \\
\text { [41] }\end{array}$ & Saudi Arabia & $\begin{array}{l}23 \\
25\end{array}$ & $\begin{array}{l}\text { PEGIFN } \propto- \\
2 \mathrm{a}+\mathrm{TDF} \\
\mathrm{TDF}\end{array}$ & Randomized controlled \\
\hline $\begin{array}{l}\text { Du Jeong, } 2017 \\
\text { [42] }\end{array}$ & Republic of Korea & 391 & TDF & Retrospective observational \\
\hline Fung, 2017 [43] & Multiple countries & $\begin{array}{l}141 \\
139\end{array}$ & $\begin{array}{l}\text { TDF } \\
\text { FTC/TDF }\end{array}$ & $\begin{array}{l}\text { Prospective, randomized, double-blind, } \\
\text { double-dummy }\end{array}$ \\
\hline Lee, 2017 [44] & Republic of Korea & $\begin{array}{l}56 \\
64\end{array}$ & $\begin{array}{l}\text { ETV } \\
\text { LAM }\end{array}$ & Phase 4, randomized \\
\hline Luo, 2017 [45] & China & $\begin{array}{l}91 \\
93\end{array}$ & $\begin{array}{l}\text { LdT } \\
\text { ETV }\end{array}$ & Prospective "real-life" \\
\hline $\begin{array}{l}\text { Rodríguez, } \\
2017[46]\end{array}$ & Spain & $\begin{array}{l}22 \\
24\end{array}$ & $\begin{array}{l}\mathrm{TDF} \\
\mathrm{LAM}+\mathrm{ADV}\end{array}$ & $\begin{array}{l}\text { Phase } 4 \text {, prospective, randomized, } \\
\text { open, controlled }\end{array}$ \\
\hline $\begin{array}{l}\text { Yang, } 2017 \\
{[47]}\end{array}$ & China & $\begin{array}{l}107 \\
115\end{array}$ & $\begin{array}{l}\text { LAM + vaccine } \\
\text { LAM + placebo }\end{array}$ & $\begin{array}{l}\text { Double-blind, randomized, placebo- } \\
\text { controlled }\end{array}$ \\
\hline Wu, 2017 [48] & Taiwan & $\begin{array}{l}106 \\
313\end{array}$ & $\begin{array}{l}\text { TDF } \\
\text { ETV }\end{array}$ & Retrospective observational \\
\hline Ahn, 2016 [49] & USA & 658 & ETV & $\begin{array}{l}\text { Observational, retrospective cohort } \\
\text { ("real world") }\end{array}$ \\
\hline Buti, 2016 [29] & Multiple countries & $\begin{array}{l}285 \\
140\end{array}$ & $\begin{array}{l}\text { TAF } \\
\text { TDF }\end{array}$ & $\begin{array}{l}\text { Randomized, double-blind, phase } 3 \text {, } \\
\text { non-inferiority }\end{array}$ \\
\hline $\begin{array}{l}\text { Chan, } 2016 \\
\text { [30] }\end{array}$ & Multiple countries & $\begin{array}{l}581 \\
292\end{array}$ & $\begin{array}{l}\text { TAF } \\
\text { TDF }\end{array}$ & $\begin{array}{l}\text { Randomized, double-blind, non- } \\
\text { inferiority }\end{array}$ \\
\hline $\begin{array}{l}\text { Huang, } 2016 \\
{[50]}\end{array}$ & China & $\begin{array}{l}79 \\
45\end{array}$ & $\begin{array}{l}\mathrm{TDF} \\
\mathrm{TDF}+\mathrm{NAs}\end{array}$ & Retrospective cohort \\
\hline Lim, 2016 [51] & Republic of Korea & $\begin{array}{l}45 \\
45\end{array}$ & $\begin{array}{l}\mathrm{TDF} \\
\mathrm{TDF}+\mathrm{ETV}\end{array}$ & Randomized open-label \\
\hline $\begin{array}{l}\text { Marcellin, } 2016 \\
\text { [52] }\end{array}$ & France & 440 & TDF & Non-interventional, prospective \\
\hline $\begin{array}{l}\text { Marcellin, } 2016 \\
\text { [53] }\end{array}$ & Multiple countries & $\begin{array}{l}185 \\
185 \\
186 \\
184\end{array}$ & $\begin{array}{l}\text { PEGIFN } \propto-2 \mathrm{a} \\
\text { TDF } \\
\text { PEGIFN } \propto- \\
2 \mathrm{a}+\text { TDF } \\
\text { PEGIFN } \propto- \\
2 \mathrm{a}+\text { TDF16 } \\
\mathrm{w}+\text { TDF alone } 32 \\
\mathrm{w}\end{array}$ & Randomized open-label, controlled \\
\hline Shen, 2016 [54] & China & $\begin{array}{l}65 \\
65\end{array}$ & $\begin{array}{l}\text { LdT } \\
\text { ETV }\end{array}$ & Prospective randomized \\
\hline $\begin{array}{l}\text { Zhang, } 2016 \\
{[55]}\end{array}$ & China & $\begin{array}{l}99 \\
97\end{array}$ & $\begin{array}{l}\text { ETV } \\
\text { LdT }\end{array}$ & Prospective cohort \\
\hline $\begin{array}{l}\text { Agarwal, } 2015 \\
\text { [56] }\end{array}$ & UK & $\begin{array}{l}10 \\
10 \\
11 \\
10 \\
10\end{array}$ & $\begin{array}{l}\text { TAF } 8 \mathrm{mg} \\
\text { TAF } 25 \mathrm{mg} \\
\text { TAF } 40 \mathrm{mg} \\
\text { TAF } 120 \mathrm{mg} \\
\text { TDF }\end{array}$ & Randomized open-label, phase $1 b$ \\
\hline
\end{tabular}


Table 1 continued

\begin{tabular}{|c|c|c|c|c|}
\hline Authors, year & Country & Patients $(n)$ & Drugs & Study design \\
\hline $\begin{array}{l}\text { Alsohaibani, } \\
2015 \text { [57] }\end{array}$ & Saudi Arabia & 68 & TDF & Retrospective, observational \\
\hline \multirow[t]{2}{*}{ Hou, 2015 [58] } & \multirow[t]{2}{*}{ China } & 257 & TDF & \multirow[t]{2}{*}{ Randomized controlled } \\
\hline & & 252 & ADV & \\
\hline Hou, 2015 [59] & China & 57 & LdT & Cohort \\
\hline \multirow{2}{*}{$\begin{array}{l}\text { Huang, } 2015 \\
{[60]}\end{array}$} & \multirow[t]{2}{*}{ China } & 33 & TDF & \multirow[t]{2}{*}{ Retrospective, observational } \\
\hline & & 65 & ETV & \\
\hline \multirow[t]{2}{*}{ Jia, 2015 [61] } & \multirow[t]{2}{*}{ China } & 68 & $\mathrm{ADV}+\mathrm{LAM}$ & \multirow[t]{2}{*}{ Case-control (prospective) } \\
\hline & & 68 & ETV & \\
\hline Kim, 2015 [62] & Republic of Korea & 52 & TDF & Retrospective observational \\
\hline \multirow[t]{2}{*}{ Kim, 2015 [63] } & \multirow[t]{2}{*}{ Republic of Korea } & 61 & LdT & \multirow[t]{2}{*}{ Retrospective observational } \\
\hline & & 90 & ETV & \\
\hline \multirow{2}{*}{$\begin{array}{l}\text { Kwon, } 2015 \\
\text { [64] }\end{array}$} & \multirow[t]{2}{*}{ Republic of Korea } & 39 & TDF & \multirow[t]{2}{*}{ Retrospective observational } \\
\hline & & 42 & ETV & \\
\hline \multirow{4}{*}{$\begin{array}{l}\text { Marcellin, } 2015 \\
\text { [65] }\end{array}$} & \multirow[t]{4}{*}{ Multiple countries } & 50 & PEGIFN $\propto-$ & \multirow[t]{4}{*}{ Randomized,open-label } \\
\hline & & 54 & $2 \mathrm{a}+\mathrm{LdT}$ & \\
\hline & & 55 & PEGIFN $\propto-2 \mathrm{a}$ & \\
\hline & & & LdT & \\
\hline \multirow{3}{*}{$\begin{array}{l}\text { Yuen, } 2015 \\
\text { [66] }\end{array}$} & \multirow[t]{3}{*}{ Republic of Korea } & 31 & Besifovir $90 \mathrm{mg}$ & \multirow[t]{3}{*}{ Randomized open-label, phase $2 b$} \\
\hline & & 28 & Besifovir $150 \mathrm{mg}$ & \\
\hline & & 30 & ETV & \\
\hline Ahn, 2014 [67] & Republic of Korea & 411 & TDF & Retrospective, observational \\
\hline \multirow[t]{2}{*}{ Berg, 2014 [68] } & \multirow[t]{2}{*}{ France, Germany, USA } & 53 & TDF & \multirow[t]{2}{*}{ Randomized, double-blind } \\
\hline & & 52 & TDF/FTC & \\
\hline Chan, 2014 & Multiple countries & 62 & TDF/FTC & Randomized, double-blind, phase 2 \\
\hline & & 64 & TDF & \\
\hline Fung, 2014 [70] & Multiple countries & 141 & TDF & Randomized, double-blind \\
\hline & & 130 & TDF/FTC & \\
\hline Jia, 2014 [71] & China & 167 & LdT & Randomized, phase 3 \\
\hline & & 165 & LAM & \\
\hline Lai, 2014 [72] & Hong Kong & & Besifovir $90 \mathrm{mg}$ & Randomized open-label, phase $2 b$ \\
\hline & & & Besifovir $150 \mathrm{mg}$ & \\
\hline & & & ETV & \\
\hline Leung,2014 & China & 16 & LdT & Randomized, open-label \\
\hline & Germany & 14 & TDF & \\
\hline & Switzerland & 16 & $\mathrm{LdT}+\mathrm{TDF}$ & \\
\hline Ozaras, 2014 & Turkey & 121 & TDF & Cohort \\
\hline & & 130 & ETV & \\
\hline Pan, 2014 [75] & USA & 90 & TDF & Open-label, single-arm, phase 4 \\
\hline Sun, 2014 [76] & China & 300 & $\mathrm{LdT}+\mathrm{ADV}$ & Randomized, open-label, controlled \\
\hline & & 299 & LdT & \\
\hline Du, 2013 [77] & China & 25 & $\mathrm{LAM}+\mathrm{ADV}$ & Prospective, randomized (pilot study) \\
\hline & & 25 & ETV & \\
\hline Gwak, 2013 & Republic of Korea & 50 & Clevudine & Comparative retrospective \\
\hline$[78]$ & & 58 & ETV & \\
\hline Hou, 2013 [79] & China & $\begin{array}{l}2600 \text { ( } 54 \text { patients excluded of } \\
\text { analysis - decompensated } \\
\text { cirrhosis) }\end{array}$ & ETV & Prospective, observational cohort \\
\hline
\end{tabular}


Table 1 continued

\begin{tabular}{|c|c|c|c|c|}
\hline Authors, year & Country & Patients $(n)$ & Drugs & Study design \\
\hline Li, 2013 [80] & China & $\begin{array}{l}14 \\
14\end{array}$ & $\begin{array}{l}\text { LAM (test) } \\
\text { LAM (branded } \\
\text { reference) }\end{array}$ & Randomized, open-label \\
\hline $\mathrm{Li}, 2013$ [81] & China & 42 & LdT & Open-label, single-arm \\
\hline Lian, 2013 [82] & China & $\begin{array}{l}60 \\
60\end{array}$ & $\begin{array}{l}\mathrm{ADV}+\mathrm{LAM} \\
\mathrm{ETV}\end{array}$ & Prospective case-control \\
\hline $\mathrm{Lu}, 2013$ [83] & China & $\begin{array}{l}30 \\
28\end{array}$ & $\begin{array}{l}\mathrm{LdT}+\mathrm{ADV} \\
\mathrm{ETV}\end{array}$ & Randomized open-label \\
\hline Luo, 2013 [84] & China & 230 & ETV & Retrospective observational \\
\hline $\begin{array}{l}\text { Marcellin, } 2013 \\
\text { [85] }\end{array}$ & Multiple countries & $\begin{array}{l}389 \\
196\end{array}$ & $\begin{array}{l}\text { TDF } \\
\text { ADV followed TDF }\end{array}$ & Randomized, open-label \\
\hline $\begin{array}{l}\text { Wang, } 2013 \\
{[86]}\end{array}$ & China & $\begin{array}{l}30 \\
25\end{array}$ & $\begin{array}{l}\mathrm{LAM}+\mathrm{ADV} \\
\mathrm{ETV}\end{array}$ & Randomized open-label \\
\hline Butti, 2012 [87] & Spain & 190 & ETV & Retrospective, observational \\
\hline Heo, 2012 [88] & Republic of Korea & $\begin{array}{l}36 \\
36\end{array}$ & $\begin{array}{l}\text { ETV } \\
\text { LAM }\end{array}$ & Randomized open-label phase 4 \\
\hline Lok, 2012 [89] & Multiple countries & $\begin{array}{l}198 \\
186\end{array}$ & $\begin{array}{l}\text { ETV + TDF } \\
\text { ETV }\end{array}$ & Randomized open-label phase $3 \mathrm{~b}$ \\
\hline $\begin{array}{l}\text { Gane, } 2011 \\
\text { [90] }\end{array}$ & Multiple countries & 389 & LdT & Open-label, single-arm \\
\hline $\begin{array}{l}\text { Patterson, } 2011 \\
\quad[91]\end{array}$ & Australia & $\begin{array}{l}38 \\
22\end{array}$ & $\begin{array}{l}\text { TDF } \\
\text { TDF + LAM }\end{array}$ & Prospective open-label \\
\hline $\begin{array}{l}\text { Perrillo, } 2011 \\
\text { [92] }\end{array}$ & Multiple countries & $\begin{array}{l}48 \\
94\end{array}$ & $\begin{array}{l}\text { LAM + placebo } \\
\mathrm{LAM}+\mathrm{ADV}\end{array}$ & Randomized open-label \\
\hline $\begin{array}{l}\text { Safadi, } 2011 \\
\text { [93] }\end{array}$ & Multiple countries & $\begin{array}{l}122 \\
124\end{array}$ & $\begin{array}{l}\text { LdT } \\
\text { LAM }\end{array}$ & Randomized, double-blind, phase $3 \mathrm{~b}$ \\
\hline Shin, 2011 [94] & Republic of Korea & $\begin{array}{l}109 \\
283\end{array}$ & $\begin{array}{l}\text { clevudine } \\
\text { ETV }\end{array}$ & Comparative retrospective \\
\hline $\begin{array}{l}\text { Wang, } 2011 \\
\text { [95] }\end{array}$ & China & $\begin{array}{l}28 \\
25\end{array}$ & $\begin{array}{l}\mathrm{LAM} \\
\mathrm{LAM}+\mathrm{ADV}\end{array}$ & Prospective controlled \\
\hline $\begin{array}{l}\text { Wang, } 2011 \\
\text { [96] }\end{array}$ & China & $\begin{array}{l}31 \\
40\end{array}$ & $\begin{array}{l}\mathrm{LAM}+\mathrm{ADV} \\
\mathrm{ETV}\end{array}$ & Prospective case-control \\
\hline Berg, 2010 [97] & Multiple countries & $\begin{array}{l}52 \\
53\end{array}$ & $\begin{array}{l}\text { FTC/TDF } \\
\text { TDF }\end{array}$ & $\begin{array}{l}\text { Randomized, double-blind, double- } \\
\text { dummy }\end{array}$ \\
\hline $\begin{array}{l}\text { Karino, } 2010 \\
\text { [98] }\end{array}$ & Japan & 82 & ETV & Open-label, single-arm \\
\hline Kim, 2010 [99] & Republic of Korea & $\begin{array}{l}24 \\
44 \\
36\end{array}$ & $\begin{array}{l}\text { ETV } \\
\text { ADV } \\
\text { ADV + LAM }\end{array}$ & Retrospective cohort \\
\hline $\begin{array}{l}\text { Kim, } 2010 \\
\quad[100]\end{array}$ & Republic of Korea & $\begin{array}{l}55 \\
73\end{array}$ & $\begin{array}{l}\text { Clevudine } \\
\text { ETV }\end{array}$ & Retrospective cohort \\
\hline Suh, 2010 [101] & $\begin{array}{l}\text { Germany } \\
\text { Republic of Korea }\end{array}$ & $\begin{array}{l}23 \\
21\end{array}$ & $\begin{array}{l}\text { LdT } \\
\text { ETV }\end{array}$ & $\begin{array}{l}\text { Open-label, parallel-group, randomized } \\
\text { (phase 3b) }\end{array}$ \\
\hline $\begin{array}{l}\text { Yokosuka, } \\
2010[8]\end{array}$ & Japan & 167 & ETV & Open-label, single-arm \\
\hline $\begin{array}{l}\text { Zheng, } 2010 \\
\text { [102] }\end{array}$ & China & $\begin{array}{l}65 \\
66\end{array}$ & $\begin{array}{l}\text { LdT } \\
\text { ETV }\end{array}$ & Open-label randomized \\
\hline $\begin{array}{c}\text { Chang, } 2009 \\
\text { [103] }\end{array}$ & Multiple countries & $\begin{array}{l}354 \\
355\end{array}$ & $\begin{array}{l}\text { ETV } \\
\text { LAM }\end{array}$ & Randomized, double-dummy \\
\hline
\end{tabular}


Table 1 continued

\begin{tabular}{|c|c|c|c|c|}
\hline Authors, year & Country & Patients $(n)$ & Drugs & Study design \\
\hline \multirow{2}{*}{$\begin{array}{l}\text { Kobashi, } 2009 \\
\text { [104] }\end{array}$} & \multirow[t]{2}{*}{ Japan } & 32 & ETV 0.1 & \multirow[t]{2}{*}{ Randomized, double-blind } \\
\hline & & 34 & ETV 0.5 & \\
\hline \multirow{2}{*}{$\begin{array}{l}\text { Liaw, } 2009 \\
\quad[105]\end{array}$} & \multirow[t]{2}{*}{ Multiple countries } & 680 & LdT & \multirow[t]{2}{*}{ Randomized, double-blind, phase 3} \\
\hline & & 687 & LAM & \\
\hline \multirow{4}{*}{$\begin{array}{l}\text { Shindo, } 2009 \\
\quad[106]\end{array}$} & \multirow[t]{4}{*}{ Japan } & 35 & ETV $0.01 \mathrm{mg}$ & \multirow[t]{4}{*}{ Randomized, double-blind } \\
\hline & & 34 & ETV $0.1 \mathrm{mg}$ & \\
\hline & & 34 & ETV $0.5 \mathrm{mg}$ & \\
\hline & & 34 & LAM $100 \mathrm{mg}$ & \\
\hline \multirow{2}{*}{$\begin{array}{c}\text { Yao, } 2009 \\
{[107]}\end{array}$} & \multirow[t]{2}{*}{ China } & 110 & Placebo/LAM & \multirow[t]{2}{*}{ Randomized, double-blind } \\
\hline & & 329 & LAM/LAM & \\
\hline \multirow{2}{*}{$\begin{array}{l}\text { Hou, } 2008 \\
\quad[108]\end{array}$} & \multirow[t]{2}{*}{ China } & 167 & LdT & \multirow[t]{2}{*}{ Randomized, double-blind } \\
\hline & & 165 & LAM & \\
\hline \multirow{2}{*}{$\begin{array}{l}\text { Marcellin, } 2008 \\
\text { [109] }\end{array}$} & \multirow[t]{2}{*}{ Multiple countries } & 426 & TDF & \multirow[t]{2}{*}{ Randomized, double-blind, phase 3} \\
\hline & & 215 & ADV & \\
\hline $\begin{array}{l}\text { Marcellin, } 2008 \\
\quad[110]\end{array}$ & Multiple countries & 65 & ADV & Open-label, single-arm * \\
\hline \multirow{2}{*}{$\begin{array}{l}\text { Sung, } 2008 \\
\text { [111] }\end{array}$} & \multirow[t]{2}{*}{ Multiple countries } & 57 & LAM & \multirow[t]{2}{*}{ Randomized, double-blind } \\
\hline & & 54 & $\mathrm{LAM}+\mathrm{ADV}$ & \\
\hline \multirow{2}{*}{$\begin{array}{l}\text { Suzuki, } 2008 \\
\quad \text { [112] }\end{array}$} & \multirow[t]{2}{*}{ Japan } & 41 & ETV 0.5 & Randomized, double-blind \\
\hline & & 43 & ETV 1.0 & \\
\hline Chan, 2007 & Multiple countries & 45 & LdT & Open-label trial \\
\hline [113] & & 44 & ADV & \\
\hline & & 46 & $\mathrm{ADV}+\mathrm{LdT}$ & \\
\hline Gish, 2007 & Multiple countries & 355 & LAM & Randomized, double-blind, double- \\
\hline [114] & & 354 & ETV & dummy \\
\hline Lai, 2007 [115] & Multiple countries & 680 & LdT & Randomized, double-blind, phase 3 \\
\hline & & 687 & LAM & \\
\hline Lim, 2007 & Caucasian & 142 & ADV & Phase 3, randomized, double-blind, \\
\hline [116] & countries & 100 & Placebo & placebo controlled \\
\hline & Asian & 138 & ADV & Phase 3, randomized, double-blind, \\
\hline & & 121 & Placebo & placebo controlled \\
\hline Rapti, 2007 & Greece & 14 & ADV & randomized controlled study \\
\hline [117] & & 28 & $\mathrm{ADV}+\mathrm{LAM}$ & \\
\hline Ren, 2007 & China & 21 & LAM & Randomized controlled \\
\hline [118] & & 21 & ETV $0.5 \mathrm{mg}$ & \\
\hline & & 19 & ETV $1.0 \mathrm{mg}$ & \\
\hline Chang, 2006 & Multiple countries & 354 & ETV & Double-blind, double-dummy, \\
\hline [119] & & 355 & LAM & randomized, controlled \\
\hline Hadziyannis, & Multiple countries & 125 & ADV & Double-blind phase \\
\hline 2006 [120] & & 62 & Placebo & $\begin{array}{l}\text { (96 weeks) }+ \text { open-label safety and } \\
\text { efficacy (144 weeks) }\end{array}$ \\
\hline Lai, 2006 [121] & Multiple countries & 325 & ETV & Randomized, double-blind, controlled \\
\hline & & 313 & LAM & \\
\hline Sherman, 2006 & Multiple countries & 141 & ETV & Randomized, double-blind, double- \\
\hline [122] & & 145 & LAM & dummy, active controlled \\
\hline Chan, 2005 & China & 50 & PEGIFN $\propto-$ & Randomized, controlled, open-label \\
\hline [123] & & 50 & $2 b+\mathrm{LAM}$ & \\
\hline & & & LAM & \\
\hline
\end{tabular}


Table 1 continued

\begin{tabular}{|c|c|c|c|c|}
\hline Authors, year & Country & Patients $(n)$ & Drugs & Study design \\
\hline $\begin{array}{l}\text { Chang, } 2005 \\
\text { [124] }\end{array}$ & Multiple countries & $\begin{array}{l}42 \\
47 \\
47 \\
45\end{array}$ & $\begin{array}{l}\text { ETV } 1.0 \\
\text { ETV } 0.5 \\
\text { ETV } 0.1 \\
\text { LAM }\end{array}$ & Randomized, dose-ranging, phase 2 \\
\hline Lai, 2005 [125] & Multiple countries & $\begin{array}{l}19 \\
22 \\
22 \\
21 \\
20\end{array}$ & $\begin{array}{l}\text { LAM } \\
\text { LdT } 400 \mathrm{mg} \\
\text { LdT } 600 \mathrm{mg} \\
\text { LAM + LdT } 400 \mathrm{mg} \\
\text { LAM + LdT } 600 \mathrm{mg}\end{array}$ & Double-blind, randomized, phase $2 \mathrm{~b}$ \\
\hline Lau, 2005 [126] & Multiple countries & $\begin{array}{l}271 \\
271 \\
272\end{array}$ & $\begin{array}{l}\text { PEGIFN } \propto-2 \mathrm{a} \\
\text { PEGIFN } \propto- \\
2 \mathrm{a}+\mathrm{LAM} \\
\text { LAM }\end{array}$ & Randomized, partially double-blind \\
\hline $\begin{array}{l}\text { Rizzetto, } 2005 \\
\text { [127] }\end{array}$ & Multiple countries & 76 & LAM & Open-label prospective \\
\hline $\begin{array}{l}\text { Sarin, } 2005 \\
\quad[128]\end{array}$ & India & $\begin{array}{l}38 \\
37\end{array}$ & $\begin{array}{l}\mathrm{IFN} \propto-2+\mathrm{LAM} \\
\mathrm{LAM}\end{array}$ & Randomized open-label \\
\hline $\begin{array}{l}\text { Liaw, 2004 } \\
\quad[129]\end{array}$ & $\begin{array}{l}\text { Asia, Australia, United } \\
\text { Kingdom }\end{array}$ & $\begin{array}{l}436 \\
215\end{array}$ & $\begin{array}{l}\text { LAM } \\
\text { Placebo }\end{array}$ & $\begin{array}{l}\text { Randomized, double-blind, placebo- } \\
\text { controlled, parallel group }\end{array}$ \\
\hline $\begin{array}{l}\text { Marcellin,2004 } \\
\quad[130]\end{array}$ & Asia, Europe & $\begin{array}{l}177 \\
179 \\
181\end{array}$ & $\begin{array}{l}\text { PEGIFN } \propto-2 \mathrm{a} \\
\text { PEGIFN } \propto- \\
2 \mathrm{a}+\text { LAM } \\
\text { LAM }\end{array}$ & Randomized, partially double-blind \\
\hline $\begin{array}{l}\text { Yao, 2004 } \\
\quad[131]\end{array}$ & China & $\begin{array}{l}322 \\
107\end{array}$ & $\begin{array}{l}\text { LAM } \\
\text { Placebo }\end{array}$ & $\begin{array}{l}\text { Randomized, double-blind, placebo } \\
\text { controlled }\end{array}$ \\
\hline Ali, 2003 [132] & Iraq & $\begin{array}{l}32 \\
30\end{array}$ & $\begin{array}{l}\text { LAM } \\
\text { Placebo }\end{array}$ & Randomized, placebo controlled \\
\hline $\begin{array}{l}\text { Dienstag, } 2003 \\
\quad[133]\end{array}$ & Multiple countries & 40 & LAM & Unblinded, observational \\
\hline $\begin{array}{l}\text { Dienstag, } 2003 \\
\quad[134]\end{array}$ & Canada, USA, England & 63 & LAM & Open label, prospective \\
\hline $\begin{array}{l}\text { Marcellin, } 2003 \\
\quad[135]\end{array}$ & Multiple countries & $\begin{array}{l}168 \\
165 \\
161\end{array}$ & $\begin{array}{l}\text { ADV } 10 \mathrm{mg} \\
\text { ADV } 30 \mathrm{mg} \\
\text { Placebo }\end{array}$ & Randomized, phase 2 \\
\hline $\begin{array}{l}\text { Schiff, } 2003 \\
\text { [136] }\end{array}$ & Multiple countries & $\begin{array}{l}119 \\
63 \\
53\end{array}$ & $\begin{array}{l}\text { LAM } \\
\text { LAM + IFN } \propto-2 b \\
\text { Placebo }\end{array}$ & Randomized, partially blinded \\
\hline Lai, 2002 [137] & Multiple countries & $\begin{array}{l}54 \\
36 \\
46 \\
41\end{array}$ & $\begin{array}{l}\text { ETV } 0.01 \mathrm{mg} \\
\text { ETV } 0.1 \mathrm{mg} \\
\text { ETV } 0.5 \mathrm{mg} \\
\text { LAM } 100 \mathrm{mg}\end{array}$ & $\begin{array}{l}\text { Randomized, double-blind, dose- } \\
\text { ranging }\end{array}$ \\
\hline Lai, 2002 [138] & China & $\begin{array}{l}50 \\
50\end{array}$ & $\begin{array}{l}\text { LAM } \\
\text { Famciclovir }\end{array}$ & Randomized, prospective \\
\hline $\begin{array}{l}\text { Mazur, } 2002 \\
\text { [139] }\end{array}$ & Poland & 45 & LAM & Open-label, prospective \\
\hline $\begin{array}{l}\text { Da Silva, } 2001 \\
\quad[140]\end{array}$ & Brazil & 32 & LAM & Open- label, prospective \\
\hline
\end{tabular}


Table 1 continued

\begin{tabular}{|c|c|c|c|c|}
\hline Authors, year & Country & Patients $(n)$ & Drugs & Study design \\
\hline $\begin{array}{l}\text { de Man, } 2001 \\
\text { [141] }\end{array}$ & Multiple countries & $\begin{array}{l}8 \\
9 \\
9 \\
8 \\
8\end{array}$ & $\begin{array}{l}\text { ETV } 0.05 \mathrm{mg} \\
\text { ETV } 0.1 \mathrm{mg} \\
\text { ETV } 0.5 \mathrm{mg} \\
\text { ETV } 1.0 \mathrm{mg} \\
\text { Placebo }\end{array}$ & $\begin{array}{l}\text { Randomized, placebo-controlled, dose- } \\
\text { escalating }\end{array}$ \\
\hline $\begin{array}{l}\text { Leung, } 2001 \\
\quad[142]\end{array}$ & China & 58 & LAM & Open-label, prospective \\
\hline $\begin{array}{l}\text { Montazeri, } \\
2001[143]\end{array}$ & Iran & $\begin{array}{l}18 \\
18\end{array}$ & $\begin{array}{l}\mathrm{LAM} \\
\mathrm{LAM}+\mathrm{IFN} \propto\end{array}$ & Randomized, open-label \\
\hline $\begin{array}{l}\text { Hadziyannis, } \\
\quad 2000[144]\end{array}$ & Greece & 25 & LAM & Open-label, single-arm, prospective \\
\hline Lau, 2000 [145] & USA & 27 & LAM & $\begin{array}{l}\text { Open-label trial, single-arm, } \\
\text { prospective }\end{array}$ \\
\hline $\begin{array}{l}\text { Liaw, } 2000 \\
\quad[146]\end{array}$ & China & $\begin{array}{l}31 \\
101 \\
41 \\
93\end{array}$ & $\begin{array}{l}\text { LAM } \\
25 \mathrm{mg}+\text { placebo } \\
\text { LAM } 25 \mathrm{mg}+\text { LAM } \\
25 \mathrm{mg} \\
\text { LAM } \\
100 \mathrm{mg}+\text { placebo } \\
\text { LAM } \\
100 \mathrm{mg}+\text { LAM } \\
100 \mathrm{mg}\end{array}$ & $\begin{array}{l}\text { Randomized, double-blind, placebo } \\
\text { controlled }\end{array}$ \\
\hline $\begin{array}{l}\text { Santantonio, } \\
\quad 2000[147]\end{array}$ & Italy & 15 & LAM & Open-label, single-arm, prospective \\
\hline $\begin{array}{l}\text { Yao, } 2000 \\
\quad[148]\end{array}$ & China & $\begin{array}{l}107 \\
322\end{array}$ & $\begin{array}{l}\text { Placebo + LAM } \\
\text { LAM + LAM }\end{array}$ & $\begin{array}{l}\text { Randomized double-blind placebo } \\
\text { controlled }\end{array}$ \\
\hline $\begin{array}{l}\text { Dienstag, } 1999 \\
\text { [149] }\end{array}$ & USA & $\begin{array}{l}66 \\
71\end{array}$ & $\begin{array}{l}\text { LAM } \\
\text { Placebo }\end{array}$ & $\begin{array}{l}\text { Prospective, randomized, double-blind, } \\
\text { placebo controlled }\end{array}$ \\
\hline $\begin{array}{l}\text { Gilson, } 1999 \\
{[150]}\end{array}$ & United Kingdom & $\begin{array}{l}15 \\
5\end{array}$ & $\begin{array}{l}\text { ADV } \\
\text { Placebo }\end{array}$ & $\begin{array}{l}\text { Randomized, double-blind, placebo } \\
\text { controlled, phase I/II }\end{array}$ \\
\hline $\begin{array}{l}\text { Tassopoulos, } \\
1999[151]\end{array}$ & Multiple countries & $\begin{array}{l}60 \\
64\end{array}$ & $\begin{array}{l}\text { LAM } \\
\text { Placebo }\end{array}$ & $\begin{array}{l}\text { Placebo controlled, double-blind, } \\
\text { randomized }\end{array}$ \\
\hline Lai, 1998 [152] & China & $\begin{array}{l}143 \\
142 \\
72\end{array}$ & $\begin{array}{l}\text { LAM } 100 \mathrm{mg} \\
\text { LAM } 25 \\
\text { Placebo }\end{array}$ & Randomized, double-blind \\
\hline Lai, 1997 [153] & China & $\begin{array}{l}12 \\
12 \\
12 \\
6\end{array}$ & $\begin{array}{l}\text { LAM } 25 \mathrm{mg} \\
\text { LAM } 100 \mathrm{mg} \\
\text { LAM } 300 \mathrm{mg} \\
\text { placebo }\end{array}$ & Randomized, placebo controlled \\
\hline $\begin{array}{l}\text { Nevens, } 1997 \\
\quad[154]\end{array}$ & Europe & $\begin{array}{l}16 \\
16 \\
19\end{array}$ & $\begin{array}{l}\text { LAM } 25 \mathrm{mg} \\
\text { LAM } 100 \mathrm{mg} \\
\text { LAM } 300 \mathrm{mg}\end{array}$ & Randomized, partially double-blind \\
\hline $\begin{array}{l}\text { Dienstag, } 1995 \\
\quad[155]\end{array}$ & USA & $\begin{array}{l}10 \\
11 \\
11\end{array}$ & $\begin{array}{l}\text { LAM } 25 \mathrm{mg} \\
\text { LAM } 100 \mathrm{mg} \\
\text { LAM } 300 \mathrm{mg}\end{array}$ & Double-blind trial \\
\hline
\end{tabular}

ADV (adefovir dipivoxil); ETV (entecavir); FTC (emtricitabine); IFN (interferon); LAM (lamivudine); LdT (telbivudine); TDF (tenofovir disoproxil fumarate); TAF (tenofovir alafenamide)

* LTSES (long-term safety and efficacy study) 
Table 2 Frequency of AEs reported according to the drug

\begin{tabular}{|c|c|c|c|c|c|c|}
\hline & LAM & ETV & LdT & ADV & TDF & TAF \\
\hline Studies & 49 & 35 & 19 & 10 & 26 & 3 \\
\hline Patients & 6419 & 5947 & 3096 & 1178 & 3566 & 876 \\
\hline AEs & 5554 & 1086 & 2302 & 1426 & 837 & 998 \\
\hline AEs/patients ${ }^{\mathrm{a}}$ & 0.87 & 0.18 & 0.74 & 1.2 & 0.23 & 1.14 \\
\hline Blood and lymphatic systems disorders & $20(0.4 \%)$ & $3(0.3 \%)$ & $22(1 \%)$ & $8(0.6 \%)$ & $9(1.1 \%)$ & - \\
\hline Cardiac disorders & $7(0.1 \%)$ & $6(0.6 \%)$ & $1(0.1 \%)$ & - & - & - \\
\hline Ear and labyrinth disorders & $5(0.1 \%)$ & $1(0.1 \%)$ & - & $1(0.1 \%)$ & - & - \\
\hline Endocrine disorders & - & - & $1(0.1 \%)$ & - & $1(0.1 \%)$ & - \\
\hline Eye disorders & - & $1(0.1 \%)$ & $6(0.3 \%)$ & $1(0.1 \%)$ & - & - \\
\hline Gastrointestinal disorders & $1116(20.1 \%)$ & $102(9.4 \%)$ & $405(17.6 \%)$ & $244(17.1 \%)$ & $128(15.3 \%)$ & $227(22.7 \%)$ \\
\hline General disorders & $811(14.6 \%)$ & $77(7.1 \%)$ & $214(9.3 \%)$ & $157(11 \%)$ & $82(9.8 \%)$ & $53(5.3 \%)$ \\
\hline Hepatobiliary disorders & $66(1.2 \%)$ & - & - & - & $9(1.1 \%)$ & - \\
\hline Infections and infestations & $871(15.7 \%)$ & $231(21.3 \%)$ & $650(28.2 \%)$ & $260(18.2 \%)$ & $110(13.1 \%)$ & $175(17.5 \%)$ \\
\hline Laboratory abnormalities & $650(11.7 \%)$ & $218(20.1 \%)$ & $347(15.1 \%)$ & $179(12.6 \%)$ & $157(18.8 \%)$ & $202(20.2 \%)$ \\
\hline Metabolism and nutrition disorders & - & - & $1(0.1 \%)$ & - & $6(0.7 \%)$ & $19(1.9 \%)$ \\
\hline Musculoskeletal and connective tissue disorders & $171(3.1 \%)$ & $24(2.2 \%)$ & $186(8.1 \%)$ & $109(7.6 \%)$ & $30(3.6 \%)$ & $25(2.5 \%)$ \\
\hline Neoplasms & $14(0.3 \%)$ & $11(1 \%)$ & $7(0.3 \%)$ & - & $7(0.8 \%)$ & - \\
\hline Nervous system disorders & $669(12 \%)$ & $193(17.8 \%)$ & $254(11 \%)$ & $216(15.1 \%)$ & $66(7.9 \%)$ & $86(8.6 \%)$ \\
\hline Psychiatric disorders & $73(1.3 \%)$ & $2(0.2 \%)$ & $1(0.1 \%)$ & - & $4(0.5 \%)$ & - \\
\hline Renal and urinary disorders & $1(0.02 \%)$ & $17(1.6 \%)$ & $2(0.1 \%)$ & $6(0.4 \%)$ & $57(6.8 \%)$ & $111(11.1 \%)$ \\
\hline Reproductive system disorders & $1(0.02 \%)$ & $1(0.1 \%)$ & - & - & - & - \\
\hline Respiratory disorders & $696(12.5 \%)$ & $19(1.7 \%)$ & $140(6.1 \%)$ & $200(14 \%)$ & $21(2.5 \%)$ & $54(5.4 \%)$ \\
\hline Skin and subcutaneous tissue disorders & $47(0.7 \%)$ & $2(0.2 \%)$ & - & & $15(1.8 \%)$ & - \\
\hline Serious AEs & $271(4.9 \%)$ & $131(12.1 \%)$ & $53(2.3 \%)$ & $39(2.7 \%)$ & $82(9.8 \%)$ & $37(3.7 \%)$ \\
\hline Drug discontinuation & $62(1.1 \%)$ & $33(3 \%)$ & $11(0.5 \%)$ & $2(0.1 \%)$ & $47(5.6 \%)$ & $9(0.9 \%)$ \\
\hline Death & $3(0.1 \%)$ & $9(0.8 \%)$ & $1(0.1 \%)$ & - & $4(0.5 \%)$ & - \\
\hline
\end{tabular}

In each column, the five AEs most often reported were scored in bold. The percentage in parentheses refers to the percentage relative to the total number of AEs reported in each drug

$A D V$ adefovir dipivoxil, AEs adverse events, ETV entecavir, $L A M$ lamivudine, $L d T$ telbivudine, TDF tenofovir disoproxil fumarate, $T A F$ tenofovir alafenamide

${ }^{a}$ Mean number of adverse events per treated patient

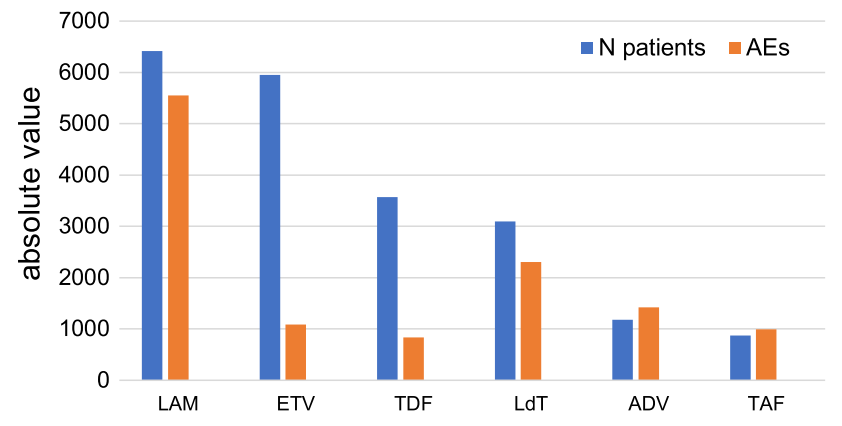

Fig. 2 Number of patients treated and absolute value of adverse events reported for each drug. $L A M$ lamivudine, ETV entecavir, TDF tenofovir disoproxil fumarate, $L d T$ telbivudine, $A D V$ adefovir dipivoxil, TAF tenofovir alafenamide
The most noticed neurological event was headache $(n=509)$. Regarding respiratory problems, viral respiratory infections were the most reported $(n=177)$.

Hepatic enzyme increase was the most documented laboratory abnormality $(n=473)$.

Although rhabdomyolysis was not described, 70 cases of elevated creatine kinase (CK) were documented, but did not lead to drug withdrawal.

\section{Entecavir (ETV)}

In the studies using ETV (0.5 or $1.0 \mathrm{mg}$ ), a total of 1086 AEs were reported (0.18 AE/patient treated) [Fig. 2, Table 2]. 
Table 3 Mean percentage decrease in hip and spine bone mineral density with TDF and TAF in studies comparing the two drugs

\begin{tabular}{llllll}
\hline Study & Follow-up & & TAF & TDF & p \\
\hline Buti, 2016 [29] & \multirow{2}{*}{48 weeks } & hip & $-0.29 \%$ & $-2.16 \%$ & $<0.0001$ \\
& & spine & $-0.88 \%$ & $-2.51 \%$ & 0.0004 \\
Chan, 2016 [30] & \multirow{2}{*}{48 weeks } & hip & $-0.1 \%$ & $-1.72 \%$ & $<0.0001$ \\
& & spine & $-0.42 \%$ & $-2.29 \%$ & $<0.0001$ \\
\hline
\end{tabular}

\begin{tabular}{llllll}
\hline Study & Follow-up & & TAF & TDF & $p$ \\
\hline Buti, 2016 [29] & \multirow{2}{*}{48 weeks } & $\uparrow \mathrm{Cr}(\mathrm{mg} / \mathrm{dl})$ & 0.01 & 0.02 & 0.32 \\
& & $\downarrow$ eGFR $(\mathrm{ml} / \mathrm{min})$ & 1.8 & 4.8 & 0.004 \\
\multirow{2}{*}{ Chan, 2016 [30] } & \multirow{2}{*}{48 weeks } & $\uparrow \mathrm{Cr}(\mathrm{mg} / \mathrm{dl})$ & 0.01 & 0.03 & 0.02 \\
& & $\downarrow$ eGFR $(\mathrm{ml} / \mathrm{min})$ & 0.6 & 5.4 & $<0.0001$
\end{tabular}

Table 4 Mean increase in serum creatinine $(\mathrm{Cr})$ from baseline and the median decrease in estimated glomerular filtration rate (eGFR) with TDF and TAF in studies comparing the two drugs
Nasopharyngitis was the most frequently described infection $(n=181)$. Regarding gastrointestinal disorders, the most common was abdominal pain $(n=115)$. Among neurological alterations, headache was the most described $(n=185)$. Regarding respiratory problems, flu syndrome was the most reported $(n=96)$.

ALT elevation was the most frequently described laboratory abnormality $(n=90)$.

CK elevation has been described in 22 patients.

\section{Tenofovir disoproxil fumarate (TDF)}

A total of 837 AEs were documented in studies with $300 \mathrm{mg}$ of TDF (0.23 AE/treated patient) [Fig. 2, Table 2].

The most frequent AEs reported were laboratory abnormalities (18.8\%), gastrointestinal disorders (15.3\%), infections and infestations (13.1\%), general disorders $(9.8 \%)$ and nervous system disorders $(7.9 \%)$.

Creatinine elevation was the most frequently described laboratory abnormality $(n=30)$. Regarding gastrointestinal disorders, the most common was nausea $(n=44)$. Nasopharyngitis was the most frequently described infection $(n=51)$. Fatigue was the most reported symptom in the general disorders section $(n=44)$.

Among neurological alterations, headache was the most described $(n=54)$. CK elevation has been described in 13 patients.

When evaluating renal and urinary disorders, 24 cases of urine erythrocytes and 2 cases of urine glucose were reported.

\section{Tenofovir alafenamide (TAF)}

A total of 998 AEs were reported in studies with $25 \mathrm{mg}$ TAF (1.14 AE/treated patient) [Fig. 2, Table 2].

The most frequent AEs reported were gastrointestinal disorders (22.7\%), laboratory abnormalities (20.2\%), 
Fig. 3 Percentage of drug discontinuation due adverse events for each nucleos(t)ide analogue. LAM lamivudine, $E T V$ entecavir, $T D F$ tenofovir disoproxil fumarate, $L d T$ telbivudine, $A D V$ adefovir dipivoxil, TAF tenofovir alafenamide

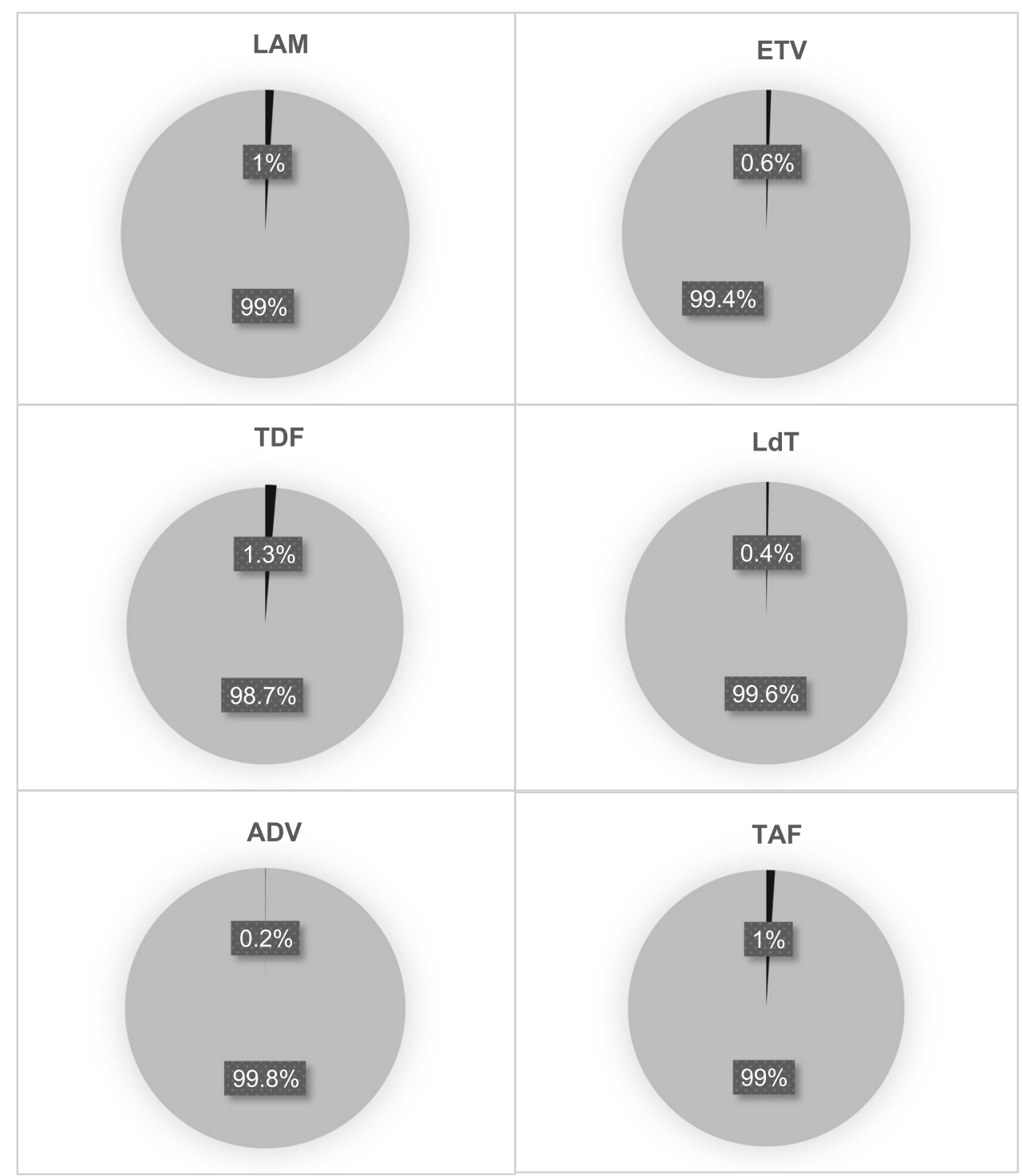

No discontinuation

Drug discontinuation infections and infestations (17.5\%), renal and urinary disorders $(11.1 \%)$ and nervous system disorders $(8.6 \%)$.

Regarding gastrointestinal disorders, the most common finding was occult blood in stool $(n=63)$. Among the laboratory abnormalities, the most reported were elevated ALT $(n=75)$ and elevated LDL cholesterol $(n=35)$. Nasopharyngitis was the main infections described $(n=89)$. Concerning the renal/urinary changes, urine erythrocytes $(n=68)$ and urine glucose $(n=43)$ were reported.

Headache was the most reported neurological disorder $(n=84)$. Elevation of $\mathrm{CK}$ has been described in 23 patients.

\section{TDF versus TAF}

Tables 3 and 4 summarized data on AEs on bone density and renal disorders, respectively, from two studies comparing TDF and TAF.

With regard to bone density, TDF caused greater bone loss in both hip and spine compared to TAF [Table 3].

On the other hand, when analysing the renal AEs, there was no clinically significant difference between the two drugs regarding the elevation of serum creatinine, but there was a greater reduction in the glomerular filtration rate in patients who received TDF [Table 4]. 


\section{Drug discontinuation due adverse events}

In the studies analysed, the percentage of drug discontinuation with LAM, ETV, TDF, LdT, ADV and TAF were, respectively, $1 \%$ (n. 62), $0.6 \%$ (n. 33), $1.3 \%$ (n. 47), $0.4 \%$ (n. 11), $0.2 \%(n .2)$ and $1 \%$ (n. 9) [Fig. 3].

\section{Discussion}

The aim of CHB treatment was to control viral replication, thereby reducing the risk of complications such as liver failure, cirrhosis and hepatocellular carcinoma. CHB treatment is often based on long-term NAs use, with the following drugs being approved: LAM, ETV, LdT, ADV, TDF and TAF, of which ETV, TDF and TAF are considered to be first-line drugs, due to its potency and high genetic barrier to resistance. Identification of potential associated AEs, even if with low incidence, might be a key factor in improving adherence and outcomes. We performed a systematic literature review of studies that included LAM, ETV, LdT, ADV, TDF and TAF since 1990 and extracted all of the reported AEs from them.

One must be aware upon reading this review, there is no necessarily causation between documented AEs and pharmacological treatment [11]. As hepatitis B infection itself may lead to extrahepatic organ involvement [12], it might be difficult to determine whether extrahepatic manifestations/symptoms are treatment-related or a disease manifestation.

Data collected in this systematic review corroborate with the understanding that serious AEs are rare within the use of NAs. The most common AEs in all NAs assessed were abdominal pain/discomfort, nasopharyngitis/upper respiratory tract infections, fatigue and headache. These symptoms are not uncommon in the general population and, perhaps, these findings are related to their high prevalence among the general population rather than to drug treatment itself.

Extrahepatic AEs may result from mitochondrial toxic effect of NAs [13]. They suppress viral replication by the inhibition of the HBV polymerase enzyme. As NA structures are similar to natural nucleosides, some of these agents can also inhibit human mitochondrial polymerase- $\gamma$ and cause mitochondrial toxicity [12, 14, 15]. Mitochondrial toxicity was first noticed during HIV treatment with highly active antiretroviral therapy. Because NAs lead to a minimal mitochondrial polymerase- $\gamma$ inhibition, NAs associated mitochondrial toxicity cases have been rarely reported. All NAs carry a warning of mitochondrial toxicity as part of their prescribing information [12, 14, 15]. Clinical manifestations of mitochondrial toxicity include hematologic disorders, peripheral neuropathy, skeletal and cardiac myopathy, pancreatitis, hepatic failure and lactic acidosis $[13,14,16]$. Among the few AE reported in studies of this systematic review, those that could be correlated to mitochondrial toxicity are CK elevation (70 cases with LAM, 49 with ETV, 211 with LdT, 22 with ADV, 13 with TDF and 23 with TAF)—but without clinical repercussion that required drug suspension; and one case of ETV-related pancreatitis.

Tenofovir disoproxil fumarate (TDF) is a prodrug of tenofovir that was approved as a NA by the United States FDA for use in CHB infection in 2008. TDF is converted to tenofovir by hydrolysis and then phosphorylated by cellular enzymes to tenofovir diphosphate [13]. It is a highly potent inhibitor of HBV DNA replication and recommended as a first-line treatment choice in CHB by the current clinical guidelines due to the absence of drug resistance [17] [18]. Tenofovir has been shown to have a potential nephrotoxic effect in patients with HIV infection who were treated for an especially extended period. However, in clinical trials, nephrotoxicity does not seem to be a major problem in HBV monoinfection [15]. Increases in serum creatinine of $>0.5 \mathrm{mg} / \mathrm{dL}$ were reported to be detected in $1 \%$ of patient [19]. Another AE concern within TDF use is the bone mass reduction. In randomized clinical trials, a great loss of bone mineral density (BMD) had been well-described in patients with HIV infection treated with TDF [20] [21, 22]. However, tenofovir-related bone fractures were not reported in patients with HBV monoinfection [20]. The exact mechanism of bone toxicity in CHB is not clear. For example, the prevalence of BMD loss in patients receiving tenofovir was similar to those who were not exposed to tenofovir. Tenofovir was reported to be associated with a lower T score only in the hips. Additionally, in this study there was no significant correlation between duration of exposure to tenofovir and reduction in BMD at any side. Additionally, a large retrospective study in Hong Kong demonstrated that BMD reduction remains stable on a plateau from year 4 through year 7 of tenofovir treatment, for both hip and lumbar spine [23]. These data indicate that loss of bone mass is not a progressive event with the use of TDF.

A pro-drug formulation, tenofovir alafenamide (TAF), was recently launched in North America and Europe, being approved for the treatment of CHB in 2016 by the FDA (Food and Drug Administration). The pharmacokinetics of TAF leads to a 6.5-times higher intracellular concentration of the phosphorylated moiety tenofovir diphosphate, and 91\% lower serum concentration of tenofovir, compared to TDF [24-26]. Given these pharmacokinetic differences, TAF dose can be far lower: a $25-\mathrm{mg}$ once-daily dose of TAF is bioequivalent to TDF at $300-\mathrm{mg}$ once daily, in terms of tenofovir plasma. Pharmacodynamic studies suggest that the lower tenofovir concentrations in plasma 
produced by TAF translate to reduced off-target drug exposure, for example, in the kidneys and bones, with implications regarding AEs [27]. TAF is, therefore, predicted to confer the same clinical efficacy as TDF, with potential improvements in its tolerability [27, 28].

In Tables 1 and 2 of this review, we report the results of two studies comparing TDF and TAF (Buti et al. and Chan et al.) [29, 30] concerning renal and bone alteration. The study by Buti et al. had a follow-up of 3 years and Chan et al. had a follow-up of 48 weeks. Both studies suggest that the bone density reduction was greater with the use of TDF, although no drug-related fractures were described. The same occurred with glomerular filtration rate, also with a greater reduction in the groups that received TDF. With these data, we raised two main questions: (1) what is the exact clinical repercussion of these findings? (2) Will such changes remain stable or continue to progress over the years?

Interestingly, renal/urinary changes were the 4th most reported group of AEs among patients on TAF, while the 6th for TDF and the 8th and 12th for ETV and LAM, respectively. Regarding reports for TAF in this group of AEs, there were 43 cases of glycosuria (versus 2 cases with TDF) and 68 cases of urine erythrocytes (versus 24 with TDF). At this time, we do not know the clinical relevance of these findings and whether they may represent any indication of renal tubular damage. Also, the number of patients treated with TAF is markedly lower than the number of patients who received the other NAs. Yet, TAF displays the highest proportion of AEs per patient treated among NAs.

These data fortify the idea that perhaps the greater safety of TAF in relation to TDF may have been overestimated, as already mentioned in the Hill et al. meta-analysis, which compared both drugs in HIV and CHB therapy [31].

It is known that susceptibility to AEs may vary by population. Previously, cases of Fanconi syndrome due to long-term use of adefovir have been reported using adefovir, with a higher incidence in East Asian populations [32]. However, in this review, the incidence of AEs according to ethnicity could not be differentiated. We believe that the low incidence of AEs from NAs makes this differentiation difficult.

Another important point to highlight is that the efficacy of treatments for $\mathrm{CHB}$ can be affected by a number of factors, including the development of AEs and poor patient compliance. In fact, a significant number of virological breakthrough may be related to medication nonadherence [33]. Hongthanakorn et al. analysed 148 patients with $\mathrm{CHB}$ and demonstrated that $38 \%$ of patients who experienced virological breakthrough were not confirmed to have antiviral resistance mutations, suggesting that medication nonadherence may be the cause of the virological breakthrough in these patients [34].
In this review, all drugs had a small percentage of discontinuation due to AEs, which is consistent with the literature. For example, Suzuki et al. reported that $1.3 \%$ of patients who were treated with ETV discontinued NA therapy because of AEs. Another study that evaluated LAM, LdT and ETV during the 3-year period found that patients with ETV had the best adherence [35]. This result strengthens the idea of ETV as one of the first-line agents in the treatment of CHB. Nevertheless, it should be emphasized that poor adherence, often still neglected, can have a negative effect on the treatment of chronic hepatitis $\mathrm{B}$, with inadequate viral suppression, increased incidence of cirrhosis and hepatocellular carcinoma, and potential emergence of NAs-resistant [36, 37]. The situation of HBV resistance to NAs in some countries is severe and, to prevent emergence of resistance, NAs with the lowest rate of genotypic resistance should be administered (TDF, TAF or ETV) and adherence reinforced [33, 36-38].

\section{Conclusion}

Treatment of CHB with NAs is safe, with a low incidence of adverse events. The most common AEs with all drugs are abdominal pain/discomfort, nasopharyngitis/upper respiratory tract infections, fatigue and headache. TDF demonstrated a greater reduction in the glomerular filtration rate and bone density of the lumbar spine and hips when compared to TAF. Currently, the number of patients treated with TAF still is too small to consolidate that TAF is really safer than TDF.

Acknowledgements SKO would like to thank Brazilian Council for Development of Science and Technology (CNPq) for Grant PQ 308609/2018-2.

Funding No grants supported the current study.

\section{Compliance with ethical standards}

Conflict of interest The authors declare that they have no conflict of interest.

Open Access This article is licensed under a Creative Commons Attribution 4.0 International License, which permits use, sharing, adaptation, distribution and reproduction in any medium or format, as long as you give appropriate credit to the original author(s) and the source, provide a link to the Creative Commons licence, and indicate if changes were made. The images or other third party material in this article are included in the article's Creative Commons licence, unless indicated otherwise in a credit line to the material. If material is not included in the article's Creative Commons licence and your intended use is not permitted by statutory regulation or exceeds the permitted use, you will need to obtain permission directly from the copyright holder. To view a copy of this licence, visit http://creativecommons. org/licenses/by/4.0/. 


\section{References}

1. World Health Organization. 2018 [Available from https://www. who.int]. Accessed $20 \mathrm{Jul} 2019$.

2. Lo AO, Wong GL. Current developments in nucleoside/nucleotide analogues for hepatitis B. Expert Rev Gastroenterol Hepatol. 2014;8:607-22.

3. easloffice@easloffice.eu EAftSotLEa, Liver EAftSot. EASL. Clinical practice guidelines on the management of hepatitis B virus infection. J Hepatol. 2017;2017(67):370-98.

4. Terrault NA, Bzowej NH, Chang KM, et al. AASLD guidelines for treatment of chronic hepatitis B. Hepatology. 2016;63:261-83.

5. Lok AS, McMahon BJ, Brown RS, et al. Antiviral therapy for chronic hepatitis B viral infection in adults: a systematic review and meta-analysis. Hepatology. 2016;63:284-306.

6. Hosaka T, Suzuki F, Kobayashi M, et al. Long-term entecavir treatment reduces hepatocellular carcinoma incidence in patients with hepatitis B virus infection. Hepatology. 2013;58:98-107.

7. Tada $\mathrm{T}$, Kumada $\mathrm{T}$, Toyoda $\mathrm{H}$, et al. Long-term natural history of liver disease in patients with chronic hepatitis B virus infection: an analysis using the Markov chain model. J Gastroenterol. 2018;53:1196-205.

8. Yokosuka O, Takaguchi K, Fujioka S, et al. Long-term use of entecavir in nucleoside-naïve Japanese patients with chronic hepatitis B infection. J Hepatol. 2010;52:791-9.

9. Moher D, Liberati A, Tetzlaff J, et al. Preferred reporting items for systematic reviews and meta-analyses: the PRISMA statement. PLoS Med. 2009;6:e1000097.

10. Booth A, Clarke M, Dooley G, et al. The nuts and bolts of PROSPERO: an international prospective register of systematic reviews. Syst Rev. 2012;1:2.

11. Mascolo A, Scavone C, Sessa M, et al. Can causality assessment fulfill the new European definition of adverse drug reaction? A review of methods used in spontaneous reporting. Pharmacol Res. 2017;123:122-9.

12. Mak LY, Seto WK, Lai CL, et al. DNA polymerase inhibitors for treating hepatitis B: a safety evaluation. Expert Opin Drug Saf. 2016;15:383-92.

13. Fontana RJ. Side effects of long-term oral antiviral therapy for hepatitis B. Hepatology. 2009;49:S185-195.

14. Wolters LM, Niesters HG, de Man RA. Nucleoside analogues for chronic hepatitis B. Eur J Gastroenterol Hepatol. 2001;13:1499-506.

15. Fung J, Lai CL, Seto WK, et al. Nucleoside/nucleotide analogues in the treatment of chronic hepatitis B. J Antimicrob Chemother. 2011;66:2715-25.

16. Martin JL, Brown CE, Matthews-Davis N, et al. Effects of antiviral nucleoside analogs on human DNA polymerases and mitochondrial DNA synthesis. Antimicrob Agents Chemother. 1994:38:2743-9.

17. Sarin SK, Kumar M, Lau GK, et al. Asian-Pacific clinical practice guidelines on the management of hepatitis B: a 2015 update. Hepatol Int. 2016;10:1-98.

18. Lok AS, McMahon BJ. Chronic hepatitis B: update 2009. Hepatology. 2009;50:661-2.

19. Pol S, Lampertico P. First-line treatment of chronic hepatitis B with entecavir or tenofovir in 'real-life' settings: from clinical trials to clinical practice. J Viral Hepat. 2012;19:377-86.

20. Ridruejo E. Treatment of chronic hepatitis B in clinical practice with entecavir or tenofovir. World $\mathrm{J}$ Gastroenterol. 2014;20:7169-80.

21. Gill US, Zissimopoulos A, Al-Shamma S, et al. Assessment of bone mineral density in tenofovir-treated patients with chronic hepatitis B: can the fracture risk assessment tool identify those at greatest risk? J Infect Dis. 2015;211:374-82.

22. Bedimo R, Maalouf NM, Zhang S, et al. Osteoporotic fracture risk associated with cumulative exposure to tenofovir and other antiretroviral agents. AIDS. 2012;26:825-31.

23. Buti M, Tsai N, Petersen J, et al. Seven-year efficacy and safety of treatment with tenofovir disoproxil fumarate for chronic hepatitis B virus infection. Dig Dis Sci. 2015;60:1457-64.

24. Vitoria M, Hill AM, Ford NP, et al. Choice of antiretroviral drugs for continued treatment scale-up in a public health approach: what more do we need to know? J Int AIDS Soc. 2016;19:20504.

25. Imaz A, Podzamczer D. Tenofovir alafenamide, emtricitabine, elvitegravir, and cobicistat combination therapy for the treatment of HIV. Expert Rev Anti Infect Ther. 2017;15:195-209.

26. Ray AS, Fordyce MW, Hitchcock MJ. Tenofovir alafenamide: a novel prodrug of tenofovir for the treatment of human immunodeficiency virus. Antiviral Res. 2016;125:63-70.

27. Ruane PJ, DeJesus E, Berger D, et al. Antiviral activity, safety, and pharmacokinetics/pharmacodynamics of tenofovir alafenamide as 10-day monotherapy in HIV-1-positive adults. J Acquir Immune Defic Syndr. 2013;63:449-55.

28. Gotham D, Hill A, Pozniak AL. Candidates for inclusion in a universal antiretroviral regimen: tenofovir alafenamide. Curr Opin HIV AIDS. 2017;12:324-33.

29. Buti M, Gane E, Seto WK, et al. Tenofovir alafenamide versus tenofovir disoproxil fumarate for the treatment of patients with HBeAg-negative chronic hepatitis B virus infection: a randomised, double-blind, phase 3, non-inferiority trial. Lancet Gastroenterol Hepatol. 2016;1:196-206.

30. Chan HL, Fung S, Seto WK, et al. Tenofovir alafenamide versus tenofovir disoproxil fumarate for the treatment of HBeAg-positive chronic hepatitis B virus infection: a randomised, doubleblind, phase 3, non-inferiority trial. Lancet Gastroenterol Hepatol. 2016;1:185-95.

31. Hill A, Hughes SL, Gotham D, et al. Tenofovir alafenamide versus tenofovir disoproxil fumarate: is there a true difference in efficacy and safety? J Virus Erad. 2018;4:72-9.

32. Kayaaslan B, Guner R. Adverse effects of oral antiviral therapy in chronic hepatitis B. World J Hepatol. 2017;9:227-41.

33. Terrault NA, Lok ASF, McMahon BJ, et al. Update on prevention, diagnosis, and treatment of chronic hepatitis B: AASLD 2018 hepatitis B guidance. Clin Liver Dis (Hoboken). 2018;12:33-4.

34. Hongthanakorn C, Chotiyaputta W, Oberhelman K, et al. Virological breakthrough and resistance in patients with chronic hepatitis $B$ receiving nucleos(t)ide analogues in clinical practice. Hepatology. 2011;53:1854-63.

35. Chien RN, Peng CY, Kao JH, et al. Higher adherence with 3-year entecavir treatment than lamivudine or telbivudine in treatment-naïve Taiwanese patients with chronic hepatitis B. J Gastroenterol Hepatol. 2014;29:185-92.

36. Kamezaki H, Kanda T, Wu S, et al. Emergence of entecavirresistant mutations in nucleos(t)ide-naive Japanese patients infected with hepatitis B virus: virological breakthrough is also dependent on adherence to medication. Scand J Gastroenterol. 2011:46:1111-7.

37. Yuen MF, Lai CL. Treatment of chronic hepatitis B: evolution over 2 decades. J Gastroenterol Hepatol. 2011;26(Suppl 1):138-43.

38. Guo X, Wu J, Wei F, et al. Trends in hepatitis B virus resistance to nucleoside/nucleotide analogues in North China from 2009-2016: a retrospective study. Int J Antimicrob Agents. 2018;52:201-9.

39. Koike K, Suyama K, Ito H, et al. Randomized prospective study showing the non-inferiority of tenofovir to entecavir in 
treatment-naïve chronic hepatitis B patients. Hepatol Res. 2018;48:59-68.

40. An J, Lim YS, Kim GA, et al. Telbivudine versus entecavir in patients with undetectable hepatitis B virus DNA: a randomized trial. BMC Gastroenterol. 2017;17:15.

41. Al Ashgar H, Peedikayil MC, Al Quaiz M, et al. HBsAg clearance in chronic hepatitis B patients with add-on pegylated interferon alfa-2a to ongoing tenofovir treatment: a randomized controlled study. Saudi J Gastroenterol. 2017;23:190-8.

42. Du Jeong I, Jung SW, Park BR, et al. Clinical course of partial virologic response with prolonged tenofovir therapy in nuclos $(\mathrm{t})$ ides-naïve patients with chronic hepatitis B. Dig Dis Sci. 2017;62:2908-14.

43. Fung S, Kwan P, Fabri M, et al. Tenofovir disoproxil fumarate (TDF) vs emtricitabine (FTC)/TDF in lamivudine resistant hepatitis B: a 5-year randomised study. J Hepatol. 2017;66:11-8.

44. Lee KS, Kweon YO, Um SH, et al. Efficacy and safety of entecavir versus lamivudine over 5 years of treatment: a randomized controlled trial in Korean patients with hepatitis B e antigen-negative chronic hepatitis B. Clin Mol Hepatol. 2017;23:331-9.

45. Luo XD, Chen XF, Zhou Y, et al. Comparison of 208-week sequential therapy with telbivudine and entecavir in $\mathrm{HBeAg}$ positive chronic hepatitis B patients with suboptimal responses to 24 weeks of Peg-IFN $\alpha$-2a therapy: an open-labelled, randomized, controlled, "real-life" trial. J Viral Hepat. 2017;24(Suppl 1):36-42.

46. Rodríguez M, Pascasio JM, Fraga E, et al. Tenofovir. World J Gastroenterol. 2017;23:7459-69.

47. Yang FQ, Rao GR, Wang GQ, et al. Phase IIb trial of. World J Gastroenterol. 2017;23:306-17.

48. Wu IT, Hu TH, Hung CH, et al. Comparison of the efficacy and safety of entecavir and tenofovir in nucleos(t)ide analogue-naive chronic hepatitis B patients with high viraemia: a retrospective cohort study. Clin Microbiol Infect. 2017;23:464-9.

49. Ahn J, Lee HM, Lim JK, et al. Entecavir safety and effectiveness in a national cohort of treatment-naïve chronic hepatitis B patients in the US - the ENUMERATE study. Aliment Pharmacol Ther. 2016;43:134-44.

50. Huang M, Jie Y, Lin G, et al. Long-term efficacy of tenofovir disoproxil fumarate therapy in nucleos $(t) i d e-e x p e r i e n c e d$ chronic hepatitis B patients. Clin Drug Investig. 2016;36:471-8.

51. Lim YS, Yoo BC, Byun KS, et al. Tenofovir monotherapy versus tenofovir and entecavir combination therapy in adefovirresistant chronic hepatitis B patients with multiple drug failure: results of a randomised trial. Gut. 2016;65:1042-51.

52. Marcellin P, Zoulim F, Hézode C, et al. Effectiveness and safety of tenofovir disoproxil fumarate in chronic hepatitis B: A 3-year, prospective, real-world study in France. Dig Dis Sci. 2016;61:3072-83.

53. Marcellin P, Ahn SH, Ma X, et al. Combination of tenofovir disoproxil fumarate and peginterferon $\alpha$-2a increases loss of hepatitis B surface antigen in patients with chronic hepatitis B. Gastroenterology. 2016;150(134-144):e110.

54. Shen H, Ding F, Wang Z, et al. Comparison of telbivudine and entecavir therapy on nephritic function and drug resistance in patients with hepatitis B virus-related compensated cirrhosis. Cell Physiol Biochem. 2016;40:370-8.

55. Zhang Y, Hu P, Qi X, et al. A comparison of telbivudine and entecavir in the treatment of hepatitis $\mathrm{B}$ e antigen-positive patients: a prospective cohort study in China. Clin Microbiol Infect. 2016;22(287):e281-289.

56. Agarwal K, Fung SK, Nguyen TT, et al. Twenty-eight day safety, antiviral activity, and pharmacokinetics of tenofovir alafenamide for treatment of chronic hepatitis B infection. J Hepatol. 2015;62:533-40.

57. Alsohaibani F, Alturaif N, Abdulshakour A, et al. Tenofovir in the treatment of naive and refractory chronic Hepatitis B: a single center experience in Saudi Arabia. Saudi J Gastroenterol. 2015;21:295-9.

58. Hou JL, Gao ZL, Xie Q, et al. Tenofovir disoproxil fumarate vs adefovir dipivoxil in Chinese patients with chronic hepatitis B after 48 weeks: a randomized controlled trial. J Viral Hepat. 2015;22:85-93.

59. Hou JL, Xu D, Shi G, et al. Long-term telbivudine treatment results in resolution of liver inflammation and fibrosis in patients with chronic hepatitis B. Adv Ther. 2015;32:727-41.

60. Huang M, Jie Y, Shi H, et al. Comparison of the efficacy of tenofovir disoproxil fumarate and entecavir for initial treatment of patient with chronic hepatitis B in China. Int J Clin Exp Med. 2015;8:666-73.

61. Jia H, Ding F, Chen J, et al. LAM add-on ADV combination therapy or ETV monotherapy for CHB patients with suboptimal response to ADV. Ann Hepatol. 2015;14:175-80.

62. Kim HJ, Cho JY, Kim YJ, et al. Long-term efficacy of tenofovir disoproxil fumarate therapy after multiple nucleos(t)ide analogue failure in chronic hepatitis B patients. Korean J Intern Med. 2015;30:32-41.

63. Kim HR, Yim HJ, Kang S, et al. Efficacy of telbivudine compared with entecavir in hepatitis B virus-related cirrhosis: 2 year follow-up data. Liver Int. 2015;35:860-9.

64. Kwon YJ, Lee HS, Park MJ, et al. Comparison of the efficacy of tenofovir and entecavir for the treatment of nucleos $(\mathrm{t})$ ide-naïve patients with chronic hepatitis B. Niger J Clin Pract. 2015;18:796-801.

65. Marcellin P, Wursthorn K, Wedemeyer H, et al. Telbivudine plus pegylated interferon alfa- $2 \mathrm{a}$ in a randomized study in chronic hepatitis B is associated with an unexpected high rate of peripheral neuropathy. J Hepatol. 2015;62:41-7.

66. Yuen MF, Ahn SH, Lee KS, et al. Two-year treatment outcome of chronic hepatitis B infection treated with besifovir vs entecavir: results from a multicentre study. J Hepatol. 2015;62:526-32.

67. Ahn SS, Chon YE, Kim BK, et al. Tenofovir disoproxil fumarate monotherapy for nucleos(t)ide-naïve chronic hepatitis B patients in Korea: data from the clinical practice setting in a singlecenter cohort. Clin Mol Hepatol. 2014;20:261-6.

68. Berg T, Zoulim F, Moeller B, et al. Long-term efficacy and safety of emtricitabine plus tenofovir DF vs tenofovir DF monotherapy in adefovir-experienced chronic hepatitis B patients. J Hepatol. 2014;60:715-22.

69. Chan HL, Chan CK, Hui AJ, et al. Effects of tenofovir disoproxil fumarate in hepatitis $\mathrm{B}$ e antigen-positive patients with normal levels of alanine aminotransferase and high levels of hepatitis B virus DNA. Gastroenterology. 2014;146:1240-8.

70. Fung S, Kwan P, Fabri M, et al. Randomized comparison of tenofovir disoproxil fumarate vs emtricitabine and tenofovir disoproxil fumarate in patients with lamivudine-resistant chronic hepatitis B. Gastroenterology. 2014;146:980-8.

71. Jia JD, Hou JL, Yin YK, et al. Two-year results of a randomized, phase III comparative trial of telbivudine versus lamivudine in Chinese patients. Hepatol Int. 2014;8:72-82.

72. Lai CL, Ahn SH, Lee KS, et al. Phase IIb multicentred randomised trial of besifovir (LB80380) vs entecavir in Asian patients with chronic hepatitis B. Gut. 2014;63:996-1004.

73. Leung NW, Herrmann E, Lau GK, et al. Early viral kinetics with telbivudine, tenofovir or combination of both in immunotolerant patients with hepatitis B e antigen-positive chronic hepatitis B. Infect Dis Ther. 2014;3:191-202. 
74. Ozaras R, Mete B, Ceylan B, et al. First-line monotherapies of tenofovir and entecavir have comparable efficacies in hepatitis B treatment. Eur J Gastroenterol Hepatol. 2014;26:774-80.

75. Pan CQ, Trinh H, Yao A, et al. Efficacy and safety of tenofovir disoproxil fumarate in Asian-Americans with chronic hepatitis B in community settings. PLoS ONE. 2014;9:e89789.

76. Sun J, Xie Q, Tan D, et al. The 104-week efficacy and safety of telbivudine-based optimization strategy in chronic hepatitis B patients: a randomized, controlled study. Hepatology. 2014;59:1283-92.

77. Du QW, Ding JG, Sun QF, et al. Combination lamivudine and adefovir versus entecavir for the treatment of naïve chronic hepatitis B patients: a pilot study. Med Sci Monit. 2013;19:751-6.

78. Gwak GY, Eo SJ, Shin SR, et al. A comparison of clevudine and entecavir for treatment-naïve patients with chronic hepatitis B: results after 2 years of treatment. Hepatol Int. 2013;7:106-10.

79. Hou JL, Jia JD, Wei L, et al. Efficacy and safety of entecavir treatment in a heterogeneous CHB population from a 'realworld' clinical practice setting in China. J Viral Hepat. 2013;20:811-20.

80. Li X, Liu B, Sun Y, et al. Single-dose pharmacokinetic properties, bioavailability, and tolerability of two lamivudine 100-mg tablet formulations: a randomized crossover study in healthy Chinese male subjects. Clin Ther. 2013;35:1546-56.

81. Li Y, Zhang Y, Wang JP, et al. Efficacy of telbivudine treatment for hepatitis B e antigen-positive chronic hepatitis B patients with poor response to adefovir dipivoxil. J Viral Hepat. 2013;20(Suppl 1):46-51.

82. Lian JS, Zeng LY, Chen JY, et al. De novo combined lamivudine and adefovir dipivoxil therapy vs entecavir monotherapy for hepatitis B virus-related decompensated cirrhosis. World J Gastroenterol. 2013;19:6278-83.

83. Lu JJ, Liu K, Ma YJ, et al. Efficacy and safety of telbivudine plus adefovir dipivoxil combination therapy and entecavir monotherapy for HBeAg-positive chronic hepatitis B patients with resistance to adefovir dipivoxil. J Viral Hepat. 2013;20(Suppl 1):40-5.

84. Luo J, Li X, Wu Y, et al. Efficacy of entecavir treatment for up to 5 years in nucleos $(\mathrm{t})$ ide-naïve chronic hepatitis B patients in real life. Int J Med Sci. 2013;10:427-33.

85. Marcellin P, Gane E, Buti M, et al. Regression of cirrhosis during treatment with tenofovir disoproxil fumarate for chronic hepatitis B: a 5-year open-label follow-up study. Lancet. 2013;381:468-75.

86. Wang JC, He LL, Chen Q. Comparison of re-treatment outcomes of lamivudine plus adefovir or entecavir in chronic hepatitis B patients with viral relapse after cessation of adefovir. Eur Rev Med Pharmacol Sci. 2013;17:1162-6.

87. Buti M, Morillas RM, Prieto M, et al. Efficacy and safety of entecavir in clinical practice in treatment-naive Caucasian chronic hepatitis B patients. Eur J Gastroenterol Hepatol. 2012;24:535-42.

88. Heo J, Park JY, Lee HJ, et al. A 96-week randomized trial of switching to entecavir in chronic hepatitis B patients with a partial virological response to lamivudine. Antivir Ther. 2012;17:1563-70.

89. Lok AS, Trinh H, Carosi G, et al. Efficacy of entecavir with or without tenofovir disoproxil fumarate for nucleos(t)ide-naïve patients with chronic hepatitis B. Gastroenterology. 2012;143(619-628):e611.

90. Gane EJ, Wang Y, Liaw YF, et al. Efficacy and safety of prolonged 3-year telbivudine treatment in patients with chronic hepatitis B. Liver Int. 2011;31:676-84.

91. Patterson SJ, George J, Strasser SI, et al. Tenofovir disoproxil fumarate rescue therapy following failure of both lamivudine and adefovir dipivoxil in chronic hepatitis B. Gut. 2011;60:247-54.

92. Perrillo RP, Hann HW, Schiff E, et al. Extended treatment with lamivudine and adefovir dipivoxil in chronic hepatitis B patients with lamivudine resistance. Hepatol Int. 2011;5:654-63.

93. Safadi R, Xie Q, Chen Y, et al. Efficacy of switching to telbivudine in chronic hepatitis B patients treated previously with lamivudine. Liver Int. 2011;31:667-75.

94. Shin SR, Yoo BC, Choi MS, et al. A comparison of 48-week treatment efficacy between clevudine and entecavir in treatmentnaïve patients with chronic hepatitis B. Hepatol Int. 2011;5:664-70.

95. Wang Z, Wu XL, Zeng WZ, et al. Lamivudine plus adefovir is a good option for chronic hepatitis B patients with viral relapse after cessation of lamivudine treatment. Virol J. 2011;8:388.

96. Wang LC, Chen EQ, Cao J, et al. De novo combination of lamivudine and adefovir versus entecavir monotherapy for the treatment of naïve $\mathrm{HBeAg-negative} \mathrm{chronic} \mathrm{hepatitis} \mathrm{B} \mathrm{patients.}$ Hepatol Int. 2011;5:671-6.

97. Berg T, Marcellin P, Zoulim F, et al. Tenofovir is effective alone or with emtricitabine in adefovir-treated patients with chronichepatitis B virus infection. Gastroenterology. 2010;139:1207-17.

98. Karino Y, Toyota J, Kumada H, et al. Efficacy and resistance of entecavir following 3 years of treatment of Japanese patients with lamivudine-refractory chronic hepatitis B. Hepatol Int. 2010;4:414-22.

99. Kim HJ, Park JH, Park DI, et al. Rescue therapy for lamivudineresistant chronic hepatitis $\mathrm{B}$ : comparison between entecavir 1.0 mg monotherapy, adefovir monotherapy and adefovir add-on lamivudine combination therapy. J Gastroenterol Hepatol. 2010;25:1374-80.

100. Kim HJ, Park DI, Park JH, et al. Comparison between clevudine and entecavir treatment for antiviral-naïve patients with chronic hepatitis B. Liver Int. 2010;30:834-40.

101. Suh DJ, Um SH, Herrmann E, et al. Early viral kinetics of telbivudine and entecavir: results of a 12-week randomized exploratory study with patients with $\mathrm{HBeAg}$-positive chronic hepatitis B. Antimicrob Agents Chemother. 2010;54:1242-7.

102. Zheng MH, Shi KQ, Dai ZJ, et al. A 24-week, parallel-group, open-label, randomized clinical trial comparing the early antiviral efficacy of telbivudine and entecavir in the treatment of hepatitis B e antigen-positive chronic hepatitis B virus infection in adult Chinese patients. Clin Ther. 2010;32:649-58.

103. Chang TT, Chao YC, Gorbakov VV, et al. Results of up to 2 years of entecavir vs lamivudine therapy in nucleoside-naïve $\mathrm{HBeAg}$-positive patients with chronic hepatitis B. J Viral Hepat. 2009;16:784-9.

104. Kobashi H, Takaguchi K, Ikeda H, et al. Efficacy and safety of entecavir in nucleoside-naive, chronic hepatitis B patients: phase II clinical study in Japan. J Gastroenterol Hepatol. 2009;24:255-61.

105. Liaw YF, Gane E, Leung N, et al. 2-Year GLOBE trial results: telbivudine Is superior to lamivudine in patients with chronic hepatitis B. Gastroenterology. 2009;136:486-95.

106. Shindo M, Chayama K, Mochida S, et al. Antiviral activity, dose-response relationship, and safety of entecavir following 24-week oral dosing in nucleoside-naive Japanese adult patients with chronic hepatitis B: a randomized, double-blind, phase II clinical trial. Hepatol Int. 2009;3:445-52.

107. Yao GB, Zhu M, Cui ZY, et al. A 7-year study of lamivudine therapy for hepatitis B virus e antigen-positive chronic hepatitis B patients in China. J Dig Dis. 2009;10:131-7.

108. Hou J, Yin YK, Xu D, et al. Telbivudine vs lamivudine in Chinese patients with chronic hepatitis B: results at 1 year of a randomized, double-blind trial. Hepatology. 2008;47:447-54. 
109. Marcellin P, Heathcote EJ, Buti M, et al. Tenofovir disoproxil fumarate versus adefovir dipivoxil for chronic hepatitis B. N Engl J Med. 2008;359:2442-555.

110. Marcellin P, Chang TT, Lim SG, et al. Long-term efficacy and safety of adefovir dipivoxil for the treatment of hepatitis B e antigen-positive chronic hepatitis B. Hepatology. 2008;48:750-8.

111. Sung JJ, Lai JY, Zeuzem S, et al. Lamivudine compared with lamivudine and adefovir dipivoxil for the treatment of $\mathrm{HBeAg}$ positive chronic hepatitis B. J Hepatol. 2008;48:728-35.

112. Suzuki F, Toyoda J, Katano Y, et al. Efficacy and safety of entecavir in lamivudine-refractory patients with chronic hepatitis B: randomized controlled trial in Japanese patients. J Gastroenterol Hepatol. 2008;23:1320-6.

113. Chan HL, Heathcote EJ, Marcellin P, et al. Treatment of hepatitis $\mathrm{B}$ e antigen positive chronic hepatitis with telbivudine or adefovir: a randomized trial. Ann Intern Med. 2007;147:745-54.

114. Gish RG, Lok AS, Chang TT, et al. Entecavir therapy for up to 96 weeks in patients with HBeAg-positive chronic hepatitis B. Gastroenterology. 2007;133:1437-44.

115. Lai CL, Gane E, Liaw YF, et al. Telbivudine versus lamivudine in patients with chronic hepatitis B. $N$ Engl $J$ Med. 2007;357:2576-88.

116. Lim SG, Marcellin P, Tassopoulos N, Hadziyannis S, Chang TT, Tong M, et al. Clinical trial: effects of adefovir dipivoxil therapy in Asian and Caucasian patients with chronic hepatitis B. Aliment Pharmacol Ther. 2007;26:1419-28.

117. Rapti I, Dimou E, Mitsoula P, et al. Adding-on versus switchingto adefovir therapy in lamivudine-resistant $\mathrm{HBeAg}$-negative chronic hepatitis B. Hepatology. 2007;45:307-13.

118. Ren FY, Piao DM, Piao XX. A 1-year trial of entecavir treatment in patients with HBeAg-positive chronic hepatitis B. World J Gastroenterol. 2007;13:4264-7.

119. Chang TT, Gish RG, de Man R, et al. A comparison of entecavir and lamivudine for $\mathrm{HBeAg-positive} \mathrm{chronic} \mathrm{hepatitis} \mathrm{B.} \mathrm{N} \mathrm{Engl}$ J Med. 2006;354:1001-100.

120. Hadziyannis SJ, Tassopoulos NC, Heathcote EJ, et al. Longterm therapy with adefovir dipivoxil for $\mathrm{HBeAg}$-negative chronic hepatitis B for up to 5 years. Gastroenterology. 2006;131:1743-51.

121. Lai CL, Shouval D, Lok AS, et al. Entecavir vs lamivudine for patients with HBeAg-negative chronic hepatitis B. N Engl J Med. 2006;354:1011-20.

122. Sherman M, Yurdaydin C, Sollano J, et al. Entecavir for treatment of lamivudine-refractory, $\mathrm{HBeAg}$-positive chronic hepatitis B. Gastroenterology. 2006;130:2039-49.

123. Chan HL, Leung NW, Hui AY, et al. A randomized, controlled trial of combination therapy for chronic hepatitis B: comparing pegylated interferon-alpha2b and lamivudine with lamivudine alone. Ann Intern Med. 2005;142:240-50.

124. Chang TT, Gish RG, Hadziyannis SJ, et al. A dose-ranging study of the efficacy and tolerability of entecavir in Lamivudinerefractory chronic hepatitis B patients. Gastroenterology. 2005;129:1198-209.

125. Lai CL, Leung N, Teo EK, et al. A 1-year trial of telbivudine, lamivudine, and the combination in patients with hepatitis B e antigen-positive chronic hepatitis B. Gastroenterology. 2005;129:528-36.

126. Lau GK, Piratvisuth T, Luo KX, et al. Peginterferon Alfa-2a, lamivudine, and the combination for $\mathrm{HBeAg}$-positive chronic hepatitis B. N Engl J Med. 2005;352:2682-95.

127. Rizzetto M, Tassopoulos NC, Goldin RD, et al. Extended lamivudine treatment in patients with $\mathrm{HBeAg}$-negative chronic hepatitis B. J Hepatol. 2005;42:173-9.

128. Sarin SK, Kumar M, Kumar R, et al. Higher efficacy of sequential therapy with interferon-alpha and lamivudine combination compared to lamivudine monotherapy in $\mathrm{HBeAg}$ positive chronic hepatitis B patients. Am J Gastroenterol. 2005; 100:2463-71.

129. Liaw YF, Sung JJ, Chow WC, et al. Lamivudine for patients with chronic hepatitis B and advanced liver disease. N Engl J Med. 2004;351:1521-31.

130. Marcellin P, Lau GK, Bonino F, et al. Peginterferon alfa-2a alone, lamivudine alone, and the two in combination in patients with HBeAg-negative chronic hepatitis B. N Engl J Med. 2004;351:1206-17.

131. Yao GB, Cui ZY, Wang BE, et al. A 3-year clinical trial of lamivudine in treatment of patients with chronic hepatitis B. Hepatobiliary Pancreat Dis Int. 2004;3:188-93.

132. Ali HY. Trial of lamivudine in hepatitis B surface antigen carriers with persistent hepatitis B core IgM antibody. Saudi Med J. 2003;24:996-9.

133. Dienstag JL, Cianciara J, Karayalcin S, et al. Durability of serologic response after lamivudine treatment of chronic hepatitis B. Hepatology. 2003;37:748-55.

134. Dienstag JL, Goldin RD, Heathcote EJ, et al. Histological outcome during long-term lamivudine therapy. Gastroenterology. 2003; 124:105-17.

135. Marcellin P, Chang TT, Lim SG, et al. Adefovir dipivoxil for the treatment of hepatitis B e antigen-positive chronic hepatitis B. N Engl J Med. 2003;348:808-16.

136. Schiff ER, Dienstag JL, Karayalcin S, et al. Lamivudine and 24 weeks of lamivudine/interferon combination therapy for hepatitis B e antigen-positive chronic hepatitis B in interferon nonresponders. J Hepatol. 2003;38:818-26.

137. Lai CL, Rosmawati M, Lao J, et al. Entecavir is superior to lamivudine in reducing hepatitis B virus DNA in patients with chronic hepatitis B infection. Gastroenterology. 2002;123:1831-8.

138. Lai CL, Yuen MF, Hui CK, et al. Comparison of the efficacy of lamivudine and famciclovir in Asian patients with chronic hepatitis B: results of 24 weeks of therapy. J Med Virol. 2002;67:334-8.

139. Mazur W, Król F, Cianciara J, et al. A multi-center open study to determine the effect of lamivudine on HBV DNA clearance and to assess the safety of the regimen in patients with chronic hepatitis B infection. Med Sci Monit. 2002;8:CR257-CR262.

140. Da Silva LC, Pinho JR, et al. Efficacy and tolerability of longterm therapy using high lamivudine doses for the treatment of chronic hepatitis B. J Gastroenterol. 2001;36:476-85.

141. de Man RA, Wolters LM, Nevens F, et al. Safety and efficacy of oral entecavir given for 28 days in patients with chronic hepatitis B virus infection. Hepatology. 2001;34:578-82.

142. Leung NW, Lai CL, Chang TT, et al. Extended lamivudine treatment in patients with chronic hepatitis B enhances hepatitis $\mathrm{B}$ e antigen seroconversion rates: results after 3 years of therapy. Hepatology. 2001;33:1527-32.

143. Montazeri G, Malekzadeh R, Nouri N, et al. The efficacy of lamivudine alone, and in combination with interferon in the treatment of resistant chronic hepatitis B. Iran J Med Sci. 2001;26:1-9.

144. Hadziyannis SJ, Papatheodoridis GV, Dimou E, et al. Efficacy of long-term lamivudine monotherapy in patients with hepatitis B e antigen-negative chronic hepatitis B. Hepatology. 2000;32:847-51.

145. Lau DT, Khokhar MF, Doo E, et al. Long-term therapy of chronic hepatitis $\mathrm{B}$ with lamivudine. Hepatology. 2000;32:828-34.

146. Liaw YF, Leung NW, Chang TT, et al. Effects of extended lamivudine therapy in Asian patients with chronic hepatitis B. Asia Hepatitis Lamivudine Study Group. Gastroenterology. 2000;119:172-80. 
147. Santantonio T, Mazzola M, Iacovazzi T, et al. Long-term follow-up of patients with anti-HBe/HBV DNA-positive chronic hepatitis B treated for 12 months with lamivudine. J Hepatol. 2000;32:300-6.

148. Yao FY, Bass NM. Lamivudine treatment in patients with severely decompensated cirrhosis due to replicating hepatitis B infection. J Hepatol. 2000;33:301-7.

149. Dienstag JL, Schiff ER, Wright TL, et al. Lamivudine as initial treatment for chronic hepatitis B in the United States. N Engl J Med. 1999;341:1256-63.

150. Gilson RJ, Chopra KB, Newell AM, et al. A placebo-controlled phase I/II study of adefovir dipivoxil in patients with chronic hepatitis B virus infection. J Viral Hepat. 1999;6:387-95.

151. Tassopoulos NC, Volpes R, Pastore G, et al. Efficacy of lamivudine in patients with hepatitis $B$ e antigen-negative/hepatitis $B$ virus DNA-positive (precore mutant) chronic hepatitis B Lamivudine Precore Mutant Study Group. Hepatology. 1999;29:889-96.
152. Lai CL, Chien RN, Leung NW, et al. A 1-year trial of lamivudine for chronic hepatitis B. Asia Hepatitis Lamivudine Study Group. N Engl J Med. 1998;339:61-8.

153. Lai CL, Ching CK, Tung AK, et al. Lamivudine is effective in suppressing hepatitis B virus DNA in Chinese hepatitis B surface antigen carriers: a placebo-controlled trial. Hepatology. 1997;25:241-4.

154. Nevens F, Main J, Honkoop P, et al. Lamivudine therapy for chronic hepatitis B: a 6-month randomized dose-ranging study. Gastroenterology. 1997;113:1258-63.

155. Dienstag JL, Perrillo RP, Schiff ER, et al. A preliminary trial of lamivudine for chronic hepatitis B infection. N Engl J Med. 1995;333:1657-61.

Publisher's Note Springer Nature remains neutral with regard to jurisdictional claims in published maps and institutional affiliations. 